\title{
CONTROLE DE SISTEMAS LINEARES INCERTOS POR MODOS DESLIZANTES E OBSERVADOR DE ALTO GANHO SEM PEAKING
}

\author{
José Paulo Vilela Soares da Cunha* \\ jpaulo@ieee.org \\ Ramon R. Costa ${ }^{\dagger}$ \\ ramonecoep.ufrj.br
}

\author{
Liu Hsu ${ }^{\dagger}$ \\ liuecoep.ufrj.br \\ Fernando Lizarralde ${ }^{\dagger}$ \\ fernando@coep.ufrj.br
}

${ }^{*}$ Departamento de Eletrônica e Telecomunicações — Faculdade de Engenharia Universidade do Estado do Rio de Janeiro Rua São Francisco Xavier 524, sala 5036A — 20559-900 — Rio de Janeiro — RJ — Brasil

†Programa de Engenharia Elétrica - COPPE — Universidade Federal do Rio de Janeiro Cx. P. 68504 - 21945-970 — Rio de Janeiro - RJ — Brasil

\section{ABSTRACT}

A model-reference variable structure controller based on a high gain observer (HGO) is proposed and analyzed. For single-input-single-output plants with relative degree larger than one, the switching law is generated using the observed state while the modulation function in the control law is generated using signals from state variable filters. With this scheme, global exponential stability with respect to an arbitrarily small residual set is achieved. Moreover, the closed loop system shows no peaking phenomena which could degrade the performance. Experimental results illustrate the good performance obtained with the algorithm in actual conditions.

KEYWORDS: Sliding mode, Variable structure, High gain observer, Reference-Model.

ARTIGO CONVIDADO:

Versão completa e revisada de artigo apresentado no CBA-2004

Artigo submetido em 16/12/04

1a. Revisão em 27/04/05

2a. Revisão em 01/12/05

3a. Revisão em 16/12/05

Aceito sob recomendação do Ed. Assoc. Prof. Paulo Eigi Miyagi

\section{RESUMO}

Neste trabalho, propõe-se um controlador por modelo de referência e modo deslizante baseado em observador de alto ganho. Para plantas monovariáveis com grau relativo maior do que um, a lei de chaveamento é gerada utilizando-se o estado observado, enquanto que a função de modulação da lei de controle utiliza sinais gerados por filtros de estado. Mostra-se que este esquema possui estabilidade global e exponencial em relação a um conjunto residual arbitrariamente pequeno. Além disso, o sistema em malha fechada não apresenta o fenômeno de peaking que pode prejudicar o desempenho. Resultados experimentais ilustram o bom desempenho obtido pelo algoritmo em condições realistas.

PALAVRAS-CHAVE: Modo deslizante, estrutura variável, observador de alto ganho, modelo de referência.

\section{INTRODUÇÃO}

O controle a estrutura variável (variable structure control VSC) é reconhecidamente bem adequado a sistemas incertos. As estratégias VSC, como propostas originalmente, necessitam de todo o estado do sistema para sua implementação (e.g., (Utkin, 1978)), o que nem sempre é disponível devido a aspectos técnicos ou econômicos como, por exemplo, 
a impossibilidade de instalação ou de construção de alguns sensores, o custo de sensores e de sistemas de comunicação entre os sensores e o controlador. Isto justifica o desenvolvimento de estratégias baseadas na realimentação de saída, em que são medidas apenas as variáveis que devem seguir sinais de referência.

Nesse contexto, o artigo pioneiro de (Bondarev et al., 1985) introduziu o uso de observadores assintóticos (Kailath, 1980) para resolver um problema fundamental do VSC, ou seja, a realização do modo deslizante "ideal" na prática. Em sistemas reais, o modo deslizante pode não ocorrer devido a atrasos em atuadores ou sensores, que usualmente não são modelados para o projeto do controlador. A idéia chave de (Bondarev et al., 1985) é o projeto do controle para se obter o modo deslizante no estado observado do sistema nominal, tratando-se os atrasos como perturbações singulares (vide também (Utkin et al., 1999, Seção 8.3)), (Hsu, 1997, Seção 2) e (Hsu et al., 2002, Seção 1.1)).

A estabilização robusta por realimentação de saída e VSC tem motivado diversos trabalhos num passado recente, e.g., (Walcott and Żak, 1988; Emelyanov et al., 1992; Esfandiari and Khalil, 1992; Oh and Khalil, 1995; Edwards and Spurgeon, 1998). Em particular, se for desejado especificar o desempenho do sistema em malha fechada, pode-se empregar o modelo de referência, o que é bastante usual no controle adaptativo (Sastry and Bodson, 1989; Ioannou and Sun, 1996). O controle por modelo de referência e modos deslizantes já foi aplicado a sistemas lineares de uma entrada e uma saída (single-input-single-output - SISO) (Hsu and Costa, 1989; Hsu et al., 1994), a sistemas lineares de múltiplas entradas e múltiplas saídas (multi-input-multi-outputMIMO) (Tao and Ioannou, 1989; Chien et al., 1996; Hsu et al., 2002; Cunha, Hsu, Costa and Lizarralde, 2003), a sistemas não-lineares SISO (Oh and Khalil, 1997; Min and Hsu, 2000) e a sistemas não-lineares MIMO (Edwards and Spurgeon, 1996; Hsu et al., 2003).

Neste artigo propõe-se uma solução alternativa à apresentada em (Hsu et al., 1994; Hsu et al., 1997) para o controle por modelo de referência e modo deslizante para sistemas lineares SISO com grau relativo arbitrário $\left(n^{*}>1\right)$. Esta solução utiliza simultaneamente um observador de estado para a geração da superfície de deslizamento e filtros de estado para gerar as funções de modulação. O observador assintótico utilizado por (Bondarev et al., 1985) é projetado para uma planta nominal e pode não ser robusto a grandes incertezas. O algoritmo aqui proposto utiliza um observador de alto ganho (high gain observer - HGO) pois este é insensível a incertezas paramétricas e perturbações na planta (Oh and Khalil, 1997).

A principal contribuição deste artigo é a introdução de um novo mecanismo para a eliminação do fenômeno conhecido como peaking, que é um problema associado ao transitório do HGO, usualmente presente neste esquema, e que pode instabilizar o controle de sistemas não-lineares (Sussmann and Kokotović, 1991). O fenômeno de peaking também é indesejável no controle de sistemas lineares pois poderia demandar muita potência, saturar ou danificar atuadores. Mostra-se que este novo algoritmo possui estabilidade global e exponencial em relação a um conjunto residual arbitrariamente pequeno, ao passo que o controlador proposto por (Oh and Khalil, 1997), embora mais geral e aplicável a sistemas nãolineares, é apenas semi-globalmente estável.

O controlador desenvolvido em (Hsu et al., 1994), denominado VS-MRAC (variable structure model-reference adaptive controller), também possui estabilidade exponencial global e não apresenta o fenômeno peaking. No entanto, o algoritmo aqui proposto apresenta algumas vantagens dignas de nota: a solução via HGO é mais intuitiva (de certa forma, reduz o problema ao caso em que $n^{*}=1$ ), a estrutura do controlador resultante é mais simples e somente um único relé é necessário para a sua implementação. A análise rigorosa da estabilidade, de ambos controladores, é igualmente complexa.

Este artigo está organizado da seguinte forma. Após a formulação do problema, revisa-se na Seção 5 o controle de sistemas de grau relativo unitário $\left(n^{*}=1\right)$ tendo em vista desenvolver o caso mais geral $n^{*}>1$. A abordagem proposta por (Hsu and Costa, 1989) é baseada em estrutura variável e realimentação de saída sem o uso de observadores. No entanto, (Edwards and Spurgeon, 1998) empregam observadores por modo deslizante no controle de sistemas com $n^{*}=1 \mathrm{com}$ a finalidade de gerar a função de modulação da lei de controle, sem nenhuma vantagem aparente em relação à abordagem de (Hsu and Costa, 1989; Cunha, Hsu, Costa and Lizarralde, 2003; Cunha, 2004). Evita-se o uso de observadores através da aplicação de filtros de estado para a geração das funções de modulação. Esta abordagem parece mais simples e natural para sistemas incertos do que o uso de observadores, pois os filtros de estado são mais simples e não requerem a malha de alto ganho ou modo deslizante adotada em observadores para sistemas incertos, e.g., (Edwards and Spurgeon, 1996; Edwards and Spurgeon, 1998).

Nas Seções 6 a 8, propõe-se uma estratégia que evita a ocorrência de peaking no sinal de controle e nos sinais da planta, apesar de não se eliminar o peaking no HGO. Para se evitar o surgimento de peaking nestes sinais, utilizam-se filtros de estado para a geração da função de modulação da lei de controle. Os esquemas de (Emelyanov et al., 1992; Esfandiari and Khalil, 1992) são sujeitos ao fenômeno peaking pois o HGO também é utilizado na geração da função de modulação da lei VSC. Na Seção 9, o peaking é eliminado do HGO 
através do escalonamento adequado do estado estimado. A Seção 10 apresenta um exemplo de aplicação do esquema proposto a um servomecanismo e inclui resultados experimentais.

\subsection{Preliminares}

São usados os seguintes conceitos e notações:

- $\|x\|$ denota a norma Euclidiana de um vetor $x$ e $\|A\|$ denota a norma da matriz $A$ induzida pela norma Euclidiana, que é dada pelo seu maior valor singular.

- A norma $\mathcal{L}_{\infty e}$ do sinal $x(t) \in \mathbb{R}^{n}$ é definida como $\left\|x_{t}\right\|_{\infty}:=\sup _{0 \leq \tau \leq t}\|x(\tau)\|$ (Ioannou and Sun, 1996, p. 70). Quando o tempo inicial é $t_{0} \leq t$, pode-se utilizar a norma

$$
\left\|x_{t, t_{0}}\right\|_{\infty}:=\sup _{t_{0} \leq \tau \leq t}\|x(\tau)\| .
$$

- Adota-se a representação mista do domínio do tempo com o domínio da transformada de Laplace (cálculo operacional). No entanto, para se definir precisamente estas representações, serão adotadas as seguintes notações: o sinal de saída $y$ de um sistema linear e invariante no tempo com matriz de transferência $H(s)$ e entrada $u$ é dado por $H(s) u$; a convolução pura $h(t) * u(t)$, sendo $h(t)$ a resposta impulsiva de $H(s)$, será escrita eventualmente como $H(s) * u$, por simplicidade.

\section{FORMULAÇÃO DO PROBLEMA}

\subsection{Planta}

Considera-se uma planta SISO linear, invariante no tempo, observável, controlável e descrita pela equação de estado

$$
\dot{x}_{p}=A_{p} x_{p}+B_{p}[u+d(t)], \quad y=C_{p} x_{p},
$$

onde $x_{p} \in \mathbb{R}^{n}$ é o vetor de estado, $u$ é o sinal de entrada, $y$ é o sinal de saída e $d$ é uma perturbação não mensurável. $\mathrm{O}$ modelo entrada/saída é dado pela função de transferência

$$
G(s)=C_{p}\left(s I-A_{p}\right)^{-1} B_{p}=K_{p} \frac{N_{p}(s)}{D_{p}(s)},
$$

onde $K_{p}$ é o ganho de alta freqüência, $N_{p}(s)$ e $D_{p}(s)$ são polinômios mônicos. Assume-se que os valores dos parâmetros da planta são incertos, mas pertencem a conjuntos conhecidos.

\subsection{Hipóteses sobre a planta}

A planta deve satisfazer as seguintes hipóteses:
(A1) $G(s)$ é de fase mínima.

(A2) $G(s)$ é estritamente própria.

(A3) A ordem do sistema ( $n$ ) é conhecida.

(A4) O grau relativo de $G(s), n^{*}$, é conhecido.

(A5) $\mathrm{O}$ sinal de $K_{p}$ é conhecido. Por simplicidade, assumese que seja positivo.

(A6) A perturbação $d(t)$ é contínua por partes e se conhece um majorante $\bar{d}(t)$ tal que $|d(t)| \leq \bar{d}(t) \leq \bar{d}_{\text {sup }}<+\infty$, $\forall t \geq 0$.

As hipóteses (A1) e (A2) são comumente adotadas em controle adaptativo por modelo de referência (Ioannou and Sun, 1996). A hipótese (A3) serve para estabelecer a ordem dos filtros de estado (11). A hipótese (A3) poderia ser relaxada de forma a se requerer apenas um majorante para $n$, como é feito em (Ioannou and Sun, 1996, Seção 6.3.1). No entanto, isto acarretaria um aumento da ordem dos filtros de estado utilizados na estrutura do controle por modelo de referência e no aumento do número de parâmetros do controlador. A hipótese (A4) também poderia ser relaxada, no entanto, a lei de controle resultante seria mais complexa (Ioannou and Sun, 1996, Seção 6.7).

Se $K_{p}<0$, então o sinal de entrada da planta deve ser invertido para satisfazer a hipótese (A5).

\subsection{Modelo de referência}

O modelo de referência é definido por (Ioannou and Sun, 1996, Seção 6.3.1)

$$
y_{M}=W_{M}(s) r, \quad W_{M}(s)=K_{M} \frac{N_{M}(s)}{D_{M}(s)},
$$

onde $y_{M}$ é o sinal de saída, $r$ é um sinal de referência contínuo por partes e uniformemente limitado, $K_{M}>0$ é o ganho de alta freqüência do modelo de referência, $N_{M}(s)$ e $D_{M}(s)$ são polinômios Hurwitz e mônicos.

Para plantas com grau relativo unitário, a função de transferência do modelo de referência $W_{M}(s)$ deve ser estritamente real positiva (strictly positive real - SPR) (Hsu and Costa, 1989), hipótese necessária na Seção 5. Para plantas com grau relativo $n^{*}>1$, em (Hsu et al., 1994; Hsu et al., 1997) escolhe-se um polinômio mônico e Hurwitz $L(s)$ de grau $N:=n^{*}-1$ de forma que a função de transferência $W_{M}(s) L(s)$ seja SPR. Aqui a escolha do modelo de referência é mais restritiva pois se requer que $W_{M}(s) L(s)=$ $K_{M} /(s+\gamma)$, com $\gamma>0$, em virtude da estrutura do HGO e da aplicação do Lema 6 (Apêndice A) na prova se estabilidade do sistema de controle em malha fechada. Assim, para 
plantas com $n^{*}>1$, a função de transferência do modelo de referência é

$$
\begin{aligned}
W_{M}(s) & =\frac{K_{M}}{s^{n^{*}}+a_{n^{*}-1} s^{n^{*}-1}+\cdots+a_{1} s+a_{0}} \\
& =\frac{K_{M}}{L(s)(s+\gamma)} .
\end{aligned}
$$

Deve-se notar que aqui as raízes de $L(s)$ não precisam ser reais. Neste aspecto há mais liberdade no projeto do que no VS-MRAC de (Hsu et al., 1994; Hsu et al., 1997), que requer que $L(s)$ tenha apenas raízes reais, uma vez que o operador $L(s)$ é realizado loc. cit. por uma conexão em cascata de $N$ filtros de avanço de fase baseados em estrutura variável com zeros simples e reais.

\subsection{Objetivo de controle}

O objetivo é projetar uma lei de controle $u$ de forma que a saída da planta $y$ rastreie assintoticamente a saída $y_{M}$ do modelo de referência, admitindo-se um pequeno erro residual. Mais precisamente, o objetivo de controle é obter a convergência assintótica do erro de saída

$$
e(t):=y(t)-y_{M}(t),
$$

para zero ou para uma pequena vizinhança residual de zero.

\section{PARAMETRIZAÇÃO DO CONTROLE}

Se a planta e a perturbação $d(t)$ fossem perfeitamente conhecidas, então uma lei de controle que casaria o sistema em malha fechada com o modelo de referência seria dada por (Cunha, Hsu, Costa and Lizarralde, 2003)

$$
\begin{aligned}
u^{*} & =\theta^{* T} \omega-W_{d}(s) * d(t), \\
W_{d}(s) & =1-\theta_{1}^{* T} \frac{A(s)}{\Lambda(s)},
\end{aligned}
$$

onde o vetor de parâmetros é dado por

$$
\theta^{* T}=\left[\begin{array}{llll}
\theta_{1}^{* T} & \theta_{2}^{* T} & \theta_{3}^{*} & \theta_{4}^{*}
\end{array}\right],
$$

$\operatorname{com} \theta_{1}^{*}, \theta_{2}^{*} \in \mathbb{R}^{(n-1)}, \theta_{3}^{*}, \theta_{4}^{*} \in \mathbb{R}$ e o vetor regressor é dado por

$$
\omega=\left[\begin{array}{llll}
\omega_{1}^{T} & \omega_{2}^{T} & y & r
\end{array}\right]^{T},
$$

onde $\omega_{1}$ e $\omega_{2}$ são gerados pelos filtros de estado

$$
\begin{gathered}
\omega_{1}=\frac{A(s)}{\Lambda(s)} u, \quad \omega_{2}=\frac{A(s)}{\Lambda(s)} y, \\
A(s)=\left[\begin{array}{lllll}
s^{n-2} s^{n-3} & \cdots & s
\end{array}\right]^{T},
\end{gathered}
$$

onde $\Lambda(s)$ é um polinômio arbitrário, mônico, Hurwitz, de grau $n-1$ e que contém $N_{M}(s)$ como fator, i.e., $\Lambda(s)=$ $\Lambda_{0}(s) N_{M}(s)$, c.f. (Ioannou and Sun, 1996, Seção 6.3.2).

Considerando-se que não há perturbação de entrada, a função de transferência de $r$ para $y$ será $W_{M}(s)$ se $\theta_{4}^{*}=K_{p}^{-1} K_{M}$ (Ioannou and Sun, 1996, eq. (6.3.12)) e for satisfeita a seguinte equação Diofantina:

$$
\begin{aligned}
N_{p}(s) \Lambda_{0}(s) D_{M}(s) & =\left[\Lambda(s)-\theta_{1}^{* T} A(s)\right] D_{p}(s) \\
& -K_{p} N_{p}(s)\left[\theta_{2}^{* T} A(s)+\theta_{3}^{*} \Lambda(s)\right] .
\end{aligned}
$$

A lei de controle (7) é desenvolvida na literatura de controle adaptativo para plantas sem perturbação de entrada $(d(t) \equiv 0)$, e.g., (Ioannou and Sun, 1996, Seção 6.3.2). O sinal $W_{d}(s) * d(t)$ cancela a perturbação de entrada conforme é mostrado na Fig. 1 (Cunha, Hsu, Costa and Lizarralde, 2003).

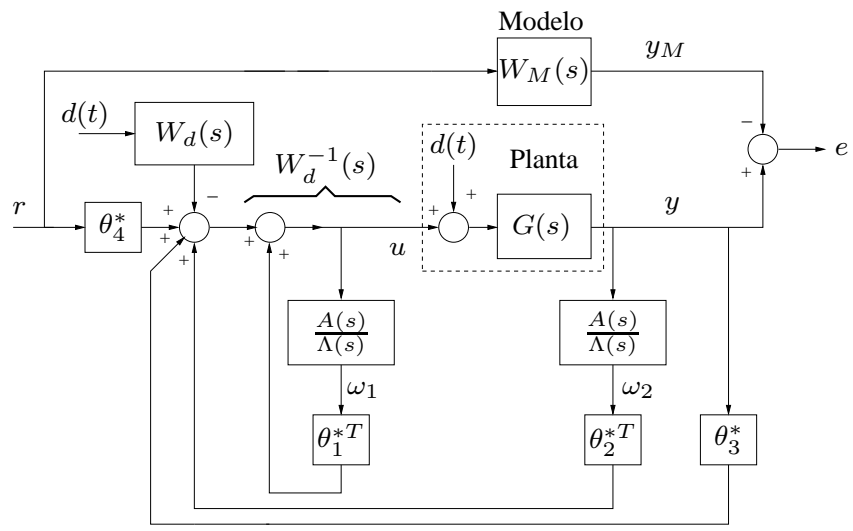

Figura 1: Estrutura do controle por modelo de referência. Destacam-se o sinal de referência $(r)$, o sinal de erro $(e)$ e o sinal $W_{d}(s) * d(t)$ para o cancelamento da perturbação $d(t)$.

\section{EQUAÇÃO DO ERRO}

Defina-se o vetor $X:=\left[x_{p}^{T}, \omega_{1}^{T}, \omega_{2}^{T}\right]^{T} \in \mathbb{R}^{3 n-2}$. Seja $\left\{A_{c}, B_{c}, C_{o}\right\}$ uma realização não-mínima de $W_{M}(s)$ com vetor de estado $X_{M}$. Então, o estado do erro $\left(X_{e}:=\right.$ $X-X_{M}$ ) e o erro de saída satisfazem (Hsu et al., 1994; Hsu et al., 2002)

$$
\begin{aligned}
\dot{X}_{e} & =A_{c} X_{e}+B_{c} K\left[u-\theta^{* T} \omega+W_{d}(s) * d(t)\right], \\
e & =C_{o} X_{e},
\end{aligned}
$$

onde $K:=\left(\theta_{4}^{*}\right)^{-1}=K_{M}^{-1} K_{p}$. O erro de saída pode ser escrito a partir de (14)-(15) como

$$
e=W_{M}(s) K\left[u-\theta^{* T} \omega+W_{d}(s) * d(t)\right] .
$$

A partir desta parametrização do controle, assume-se que 
(A7) A lei de controle satisfaz a desigualdade $\left\|u_{t, 0}\right\|_{\infty} \leq$ $k_{\omega}\left\|\omega_{t, 0}\right\|_{\infty}+k_{r d}$, onde $k_{\omega}, k_{r d}>0$.

Isto impede o escape em tempo finito dos sinais do sistema. De fato, os sinais do sistema serão regulares e, portanto, podem crescer no máximo exponencialmente (Sastry and Bodson, 1989). A hipótese (A7) não restringe a classe de sistemas que podem ser controlados, mas apenas se aplica à amplitude da lei de controle e, conseqüentemente, sobre a função de modulação da lei VSC, conforme os Teoremas 1 , 3 e 4 adiante.

\section{CONTROLE DE SISTEMAS DE GRAU RELATIVO UNITÁRIO}

Para $n^{*}=1$, tem-se que $N=n^{*}-1=0, L(s)=1$ e o modelo de referência $W_{M}(s)$ pode ser escolhido SPR. Assim, a partir da equação do erro (16) e de acordo com o Lema 5 (Apêndice A), a lei de controle a estrutura variável por modelo de referência (VS-MRAC) originalmente proposta em (Hsu and Costa, 1989) é dada por (Hsu et al., 1994)

$$
\begin{aligned}
u & =u^{\mathrm{nom}}+U, \quad U=-\rho \operatorname{sgn}(e), \\
u^{\mathrm{nom}} & =\left(\theta^{\mathrm{nom}}\right)^{T} \omega,
\end{aligned}
$$

onde $\theta^{\text {nom }}$ é o valor nominal de $\theta^{*}$. O controle nominal $u^{\text {nom }}$ permite a redução da amplitude da função de modulação $\rho$ se a incerteza $\left\|\theta^{*}-\theta^{\text {nom }}\right\|$ for pequena.

A aplicação do Lema 5 (Apêndice A) permite concluir que o algoritmo acima é exponencialmente estável desde que a função de modulação $\rho \in \mathbb{R}^{+}$satisfaça a desigualdade

$$
\rho \geq \delta+\left|\left(\theta^{\text {nom }}-\theta^{*}\right)^{T} \omega+W_{d}(s) * d(t)\right|,
$$

onde $\delta \geq 0$ é uma constante arbitrária. Uma função de modulação que satisfaz a desigualdade (19) e a hipótese (A7) é

$$
\rho=\delta+c_{1}\|\omega\|+\hat{d}(t)
$$

com

$\hat{d}(t)=\bar{d}(t)+\frac{c_{2}}{s+\gamma_{d}} * \bar{d}(t) \quad\left(\geq\left|\left(1-\theta_{1}^{* T} \frac{A(s)}{\Lambda(s)}\right) * d(t)\right|\right)$,

onde $c_{1} \geq\left\|\theta^{*}-\theta^{\text {nom }}\right\|$. O majorante (21) é obtido pela aplicação do Lema 7 (Apêndice A), de onde se tem que $c_{2} \geq 0$ é uma constante apropriada e $\gamma_{d}$ satisfaz $\gamma_{0}>\gamma_{d}>0$, sendo $\gamma_{0}$ a margem de estabilidade do filtro com matriz de transferência $A(s) \Lambda^{-1}(s)$, c.f. (69). Alguns métodos para a computação dos coeficientes $c_{2}$ e $\gamma_{d}$ do filtro (21) são propostos em (Cunha, Costa and Hsu, 2003). À frente, na Seção 10.2, é apresentado um exemplo de projeto da função de modulação.
O seguinte resultado de estabilidade foi provado originalmente em (Hsu and Costa, 1989):

Teorema 1 Para $n^{*}=1$, considere-se o sistema formado pela equação do erro (14)-(15) e pela lei de controle (17) (18). Se as hipóteses (A1)-(A6) forem satisfeitas e a função de modulação satisfizer a hipótese (A7) e a desigualdade (19), então a estratégia VS-MRAC é globalmente exponencialmente estável, i.e., $\exists k, \lambda>0$ tais que $\left\|X_{e}(t)\right\| \leq$ $k e^{-\lambda t}\left\|X_{e}(0)\right\|, \forall t \geq 0$. Além disso, se $\delta>0$, então o erro de saída e $(t)$ se tornará nulo após algum tempo finito.

Prova: Aplica-se o Lema 5 ao sistema composto pela realização não-mínima da equação (16), dada pela equação do erro (14)-(15), e pela lei de controle (17)-(18).

No esquema do VS-MRAC na Fig. 2 foi assinalado o laço do modo deslizante "ideal", no qual a função de transferência do sinal $U$ para o sinal $e$ é de fase mínima e possui grau relativo unitário, o que possibilita a ocorrência do modo deslizante na superfície de deslizamento $e=0$ (Utkin et al., 1999, Seção 8.3).

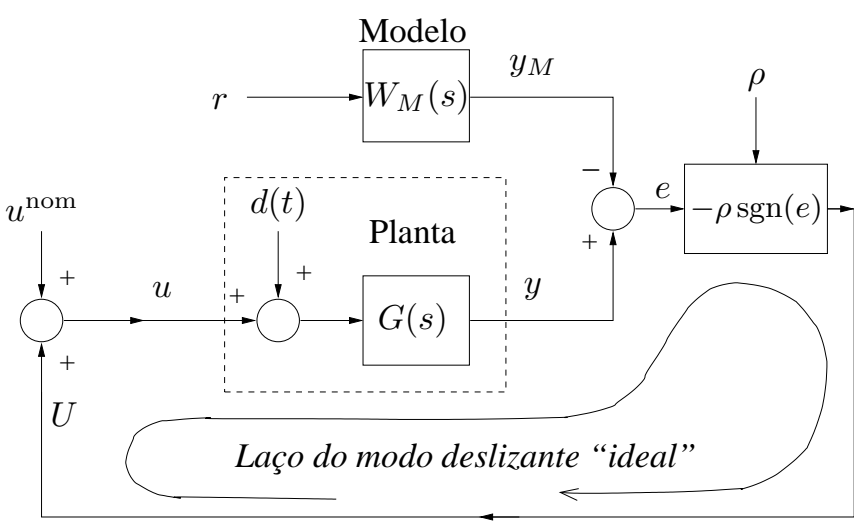

Figura 2: Controlador por modelo de referência de sistemas com grau relativo $n^{*}=1$. Os filtros de estado e a síntese da função de modulação $\rho$ foram omitidos para simplificar o diagrama.

\section{CONTROLE DE SISTEMAS DE GRAU RELATIVO SUPERIOR A UM}

Para $n^{*}>1$, o controle à estrutura variável baseado em realimentação de saída não pode ser aplicado diretamente, uma vez que o modelo de referência não é SPR. A abordagem VSMRAC de (Hsu et al., 1994; Hsu et al., 1997) é baseada no erro de predição e em filtros de avanço de fase por estrutura variável. 
Aqui se propõe uma estratégia de controle alternativa baseada em um HGO, que parece ser mais simples do que o VSMRAC. Uma idéia natural seria estimar o estado da planta como no controlador para estabilização robusta em $(\mathrm{Oh}$ and Khalil, 1995). No entanto, aqui se utiliza o modelo de referência (sem incerteza) em vez do modelo da planta (incerto) para reconstruir o estado da equação do erro, adotando-se o argumento de (Chang and Lee, 1996) de que é mais fácil construir um observador para o modelo de referência do que para a planta. Uma abordagem similar a esta é proposta por (Oh and Khalil, 1997) para o rastreamento de trajetórias em sistemas não-lineares. No entanto, tanto (Oh and Khalil, 1995) quanto (Oh and Khalil, 1997) utilizam uma lei VSC em que a função de modulação é globalmente limitada a fim de se eliminar o fenômeno peaking, mas pagando-se o preço de que as propriedades de estabilidade são semi-globais.

Aqui se utiliza um HGO para gerar a lei de chaveamento e, ao mesmo tempo, se utiliza uma função de modulação gerada por filtros de estado para se obter a estabilidade global sem que o fenômeno peaking se manifeste na planta e no sinal de comando, embora o peaking ocorra no HGO.

Considere-se a realização $\left\{A_{M}, B_{M}, C_{M}\right\}$ de ordem mínima na forma canônica do observador para o modelo de referência $W_{M}(s)$ dada pelas matrizes

$$
\begin{aligned}
A_{M} & =\left[\begin{array}{cccc}
-a_{n^{*}-1} & 1 & \cdots & 0 \\
\vdots & & \ddots & \vdots \\
-a_{1} & 0 & & 1 \\
-a_{0} & 0 & \cdots & 0
\end{array}\right], \quad B_{M}=\left[\begin{array}{c}
0 \\
\vdots \\
0 \\
K_{M}
\end{array}\right], \\
C_{M} & =\left[\begin{array}{llll}
1 & 0 & \cdots & 0
\end{array}\right] .
\end{aligned}
$$

Então, a equação do erro (14)-(15) pode ser reescrita como

$$
\begin{aligned}
\dot{x}_{e} & =A_{M} x_{e}+B_{M} K\left[u-\theta^{* T} \omega+W_{d}(s) * d(t)+\pi_{e}\right], \\
e & =C_{M} x_{e},
\end{aligned}
$$

onde a condição inicial $x_{e}(0)$ e o sinal escalar exponencialmente decrescente $\pi_{e}(t)$ são adequados para representar os efeitos das condições iniciais dos modos observáveis mas não-controláveis na equação do erro (14)-(15). O sinal $\pi_{e}(t)$ satisfaz $\left|\pi_{e}(t)\right| \leq k_{e} e^{-\lambda_{e} t}\left\|X_{e}(0)\right\|, \forall t \geq 0$, com constantes apropriadas $k_{e}, \lambda_{e}>0$. A Observação 1 na Seção 7.1 justifica a inclusão do sinal $\pi_{e}(t)$ na equação dinâmica em vez de se acrescentar um sinal exponencialmente decrescente na equação de saída para representar os efeitos das condições iniciais dos modos observáveis mas não-controláveis, como poderia parecer mais natural.

É possível projetar uma matriz $S \in \mathbb{R}^{1 \times n^{*}}$ que define a superfície de chaveamento ideal $\sigma\left(x_{e}\right)=0$, onde $\sigma\left(x_{e}\right):=S x_{e}$, para que $\left\{A_{M}, B_{M}, S\right\}$ seja uma realização da função de transferência SPR $W_{M}(s) L(s)$. Tendo em vista o modelo de referência (5) e as matrizes (22), tem-se (Kailath, 1980, pp. 39-45)

$$
S^{T}=\left[\begin{array}{ccccc}
0 & \ldots & 0 & 0 & 1 \\
0 & \ldots & 0 & 1 & a_{n^{*}-1} \\
\vdots & . \cdot & 1 & a_{n^{*}-1} & a_{n^{*}-2} \\
0 & . \cdot & . \cdot & & \vdots \\
1 & a_{n^{*}-1} & a_{n^{*}-2} & \ldots & a_{1}
\end{array}\right]^{-1}\left[\begin{array}{c}
1 \\
l_{N-1} \\
\vdots \\
l_{1} \\
l_{0}
\end{array}\right],
$$

onde $l_{i}$ são os coeficientes do polinômio $L(s)=s^{N}+$ $l_{N-1} s^{N-1}+\cdots+l_{1} s+l_{0}$ e $N=n^{*}-1$.

Assumindo-se que uma estimativa $\hat{x}_{e}$ de $x_{e}$ esteja disponível, então uma lei de controle é

$$
u=u^{\mathrm{nom}}+U, \quad U=-\rho \operatorname{sgn}\left(S \hat{x}_{e}\right),
$$

onde o controle nominal $u^{\text {nom }}$ é dado por (18). Se $\hat{x}_{e}(t) \equiv$ $x_{e}(t)$ e a função de modulação $\rho$ satisfizer a desigualdade (19) então o estado $x_{e}$ e o erro de saída $e$ convergem exponencialmente para zero, como se pode concluir através da aplicação do Lema 6 (Apêndice A) $\operatorname{com} \beta(t) \equiv 0$.

\section{OBSERVADOR DE ALTO GANHO}

Como o estado $x_{e}$ não é disponível, a lei de controle utilizará o estado $\hat{x}_{e}$ estimado pelo $\mathrm{HGO}$

$$
\begin{aligned}
\dot{\hat{x}}_{e} & =A_{M} \hat{x}_{e}+B_{M} K^{\mathrm{nom}} U-\left[\alpha\left(\varepsilon^{-1}\right)-a_{M}\right] \tilde{e}, \\
\tilde{e} & =C_{M} \hat{x}_{e}-e,
\end{aligned}
$$

onde e é e erro de saída do observador, $K^{\text {nom }}$ é o valor nominal do ganho $K$ e o vetor

$$
a_{M}=\left[\begin{array}{llll}
a_{n^{*}-1} & \cdots & a_{1} & a_{0}
\end{array}\right]^{T} .
$$

Os coeficientes $\alpha_{i}$ no vetor de realimentação do observador (Lu and Spurgeon, 1998)

$$
\alpha\left(\varepsilon^{-1}\right)=\left[\begin{array}{llll}
\frac{\alpha_{n^{*}}-1}{\varepsilon} & \cdots & \frac{\alpha_{1}}{\varepsilon^{n^{*}-1}} & \frac{\alpha_{0}}{\varepsilon^{n^{*}}}
\end{array}\right]^{T}
$$

devem ser escolhidos de forma que o polinômio característico do observador em malha fechada seja Hurwitz, o que se obtém se o polinômio

$$
N_{\alpha}(s)=s^{n^{*}}+\alpha_{n^{*}-1} s^{n^{*}-1}+\cdots+\alpha_{0}
$$

for Hurwitz e $\varepsilon>0$. Para que as incertezas e perturbações no sistema afetem pouco o estado estimado $\hat{x}_{e}$, a norma do ganho da realimentação do observador $\left(\left\|\alpha\left(\varepsilon^{-1}\right)\right\|\right)$ deve ser alta, o que requer que $\varepsilon$ seja pequeno. 


\subsection{Majorante do erro de estimação}

A seguir, o Teorema 2 estabelece majorantes para a norma do erro de estimação do estado $\tilde{x}_{e}(t):=\hat{x}_{e}(t)-x_{e}(t)$ do observador de alto ganho, tendo em vista a prova de estabilidade do sistema de controle em malha fechada.

Para simplificar este Teorema, assume-se que a margem de estabilidade do polinômio $N_{\alpha}(s)\left(\bar{\lambda}_{\alpha}:=\min _{j}\left\{-\Re\left(z_{j}\right)\right\}\right.$, onde $\left\{z_{j}\right\}$ são as raízes de $\left.N_{\alpha}\left(z_{j}\right)=0\right)$ e o escalar $\lambda_{\alpha}$ satisfaçam a desigualdade $0<\lambda_{e}<\lambda_{\alpha}<\bar{\lambda}_{\alpha}$. Também se impõe que $0<\varepsilon \leq 1$.

Teorema 2 Considere o observador (26)-(29) e a equação do erro (14)-(15). Se a hipótese (A7) for satisfeita, os sinais $r(t)$ e $d(t)$ forem uniformemente limitados, o polinômio $N_{\alpha}(s)$ for Hurwitz e o parâmetro $\varepsilon$ for mantido na faixa $0<\varepsilon \leq 1$, então $\exists k_{1}, \ldots, k_{6}>0$ tais que o erro de estimação do estado $\left(\tilde{x}_{e}\right)$ satisfaz as desigualdades

$$
\begin{aligned}
\left\|\tilde{x}_{e}(t)\right\| & \leq \frac{k_{1}}{\varepsilon^{n^{*}-1}}\left\|\tilde{x}_{e}(0)\right\| e^{-\frac{\lambda_{\alpha}}{\varepsilon} t}+k_{2} \varepsilon\left\|X_{e}(0)\right\| e^{-\lambda_{e} t} \\
& +e^{-\frac{\lambda_{\alpha}}{\varepsilon} t} *\left[k_{3}\|\omega(t)\|+k_{4}\right] \\
\left\|\tilde{x}_{e}(t)\right\| & \leq \frac{k_{1}}{\varepsilon^{n^{*}-1}}\left\|\tilde{x}_{e}(0)\right\| e^{-\frac{\lambda_{\alpha}}{\varepsilon} t}+k_{2} \varepsilon\left\|X_{e}(0)\right\| e^{-\lambda_{e} t} \\
& +\varepsilon C(t, 0)
\end{aligned}
$$

$\forall t \geq 0$, onde

$$
C\left(t, t_{0}\right)=k_{5}\left\|\omega_{t, t_{0}}\right\|_{\infty}+k_{6}
$$

Prova: Reescreve-se a equação do erro de saída do observador (27) como $\tilde{e}=C_{M} \tilde{x}_{e}$. Subtraindo-se (23) de (26) se obtém a equação da dinâmica do erro de estimação do estado

$$
\dot{\tilde{x}}_{e}=A_{e}\left(\varepsilon^{-1}\right) \tilde{x}_{e}+B_{M}\left[K^{n o m} \bar{U}-K \pi_{e}(t)\right],
$$

onde são utilizados a matriz

$$
\begin{aligned}
A_{e}\left(\varepsilon^{-1}\right)= & A_{M}-\left[\alpha\left(\varepsilon^{-1}\right)-a_{M}\right] C_{M} \\
= & {\left[\begin{array}{ccccc}
-\frac{\alpha_{n^{*}-1}}{\alpha_{n^{*}}-2} & 1 & 0 & \cdots & 0 \\
\varepsilon^{2} & 0 & 1 & & 0 \\
\vdots & \vdots & & \ddots & \vdots \\
-\frac{\alpha_{1}}{\varepsilon^{n^{*}-1}} & 0 & 0 & & 1 \\
-\frac{\alpha_{0}}{\varepsilon^{n^{*}}} & 0 & 0 & \cdots & 0
\end{array}\right] . }
\end{aligned}
$$

e o sinal

$$
\begin{aligned}
\bar{U} & :=\left[1-\left(K^{\mathrm{nom}}\right)^{-1} K\right] U+\left(K^{\mathrm{nom}}\right)^{-1} K \\
& \times\left[\left(\theta^{*}-\theta^{\mathrm{nom}}\right)^{T} \omega-W_{d}(s) * d(t)\right] .
\end{aligned}
$$

Então, aplicando-se a transformação

$$
\bar{x}_{e}=T(\varepsilon) \tilde{x}_{e}, \quad T(\varepsilon)=\operatorname{diag}\left\{1, \varepsilon, \ldots, \varepsilon^{n^{*}-1}\right\},
$$

reescreve-se a equação do erro (34) como

$$
\varepsilon \dot{\bar{x}}_{e}=\bar{A}_{e} \bar{x}_{e}+\varepsilon \bar{B}_{M}(\varepsilon)\left[K^{n o m} \bar{U}-K \pi_{e}(t)\right],
$$

onde $\bar{A}_{e}$ é uma matriz constante na forma companheira com polinômio característico $N_{\alpha}(s), \bar{B}_{M}(\varepsilon)=$ $\left[0, \ldots, 0, \varepsilon^{n^{*}-1}\right]^{T} K_{M}$ e, conseqüentemente, $\left\|\bar{B}_{M}(\varepsilon)\right\|=$ $\varepsilon^{n^{*}-1} K_{M}$. Assim sendo, a norma do estado $\bar{x}_{e}$ pode ser majorada por

$$
\begin{aligned}
\left\|\bar{x}_{e}(t)\right\| & \leq k_{1}\left\|\bar{x}_{e}(0)\right\| e^{-\frac{\lambda_{\alpha}}{\varepsilon} t}+k_{2} \varepsilon^{n^{*}}\left\|X_{e}(0)\right\| e^{-\lambda_{e} t} \\
& +k_{7} \frac{\varepsilon^{n^{*}}}{\varepsilon s+\lambda_{\alpha}} *\|\bar{U}(t)\|, \quad \forall t \geq 0 .
\end{aligned}
$$

Uma vez que a hipótese (A7) é satisfeita e os sinais $r(t)$ e $d(t)$ são uniformemente limitados, conclui-se a partir de (36) que $\exists k_{\bar{U} \omega}, k_{\bar{U}} \geq 0$ tais que $\left\|\bar{U}_{t, 0}\right\|_{\infty} \leq k_{\bar{U} \omega}\left\|\omega_{t, 0}\right\|_{\infty}+k_{\bar{U}}$, $\forall t \geq 0$. Por outro lado, a partir da definição da transformação (37), tem-se que $\|T(\varepsilon)\|=1$ e $\left\|T^{-1}(\varepsilon)\right\|=\varepsilon^{1-n^{*}}$, uma vez que $\varepsilon \in(0,1]$. Utilizando-se estes fatos, obtém-se a partir de (39) os majorantes (31) e (32) para a norma do erro de estimação do estado.

Observação 1 Poderia parecer mais natural representar os efeitos das condições iniciais dos modos observáveis mas não-controláveis da equação do erro (14)-(15) através da inclusão de um sinal exponencialmente decrescente $\bar{\pi}_{e}(t)$ na equação de saída (23), que passaria a ser

$$
e=C_{M} x_{e}+\bar{\pi}_{e}(t)
$$

e permitiria a eliminação do sinal $\pi_{e}(t)$ da equação (23). No entanto, a inclusão do termo $\bar{\pi}_{e}(t)$ resultaria no majorante

$$
\begin{aligned}
\left\|\tilde{x}_{e}(t)\right\| & \leq \frac{k_{1}}{\varepsilon^{n^{*}-1}}\left\|\tilde{x}_{e}(0)\right\| e^{-\frac{\lambda_{\alpha}}{\varepsilon} t}+\frac{\bar{k}_{2}}{\varepsilon^{n^{*}-1}}\left\|X_{e}(0)\right\| e^{-\lambda_{e} t} \\
& +e^{-\frac{\lambda_{\alpha}}{\varepsilon} t} *\left[k_{3}\|\omega(t)\|+k_{4}\right],
\end{aligned}
$$

onde se nota que o termo $\frac{\bar{k}_{2}}{\varepsilon^{n^{*}-1}}\left\|X_{e}(0)\right\| e^{-\lambda_{e} t}$ é muito mais conservador do que o termo $k_{2} \varepsilon\left\|X_{e}(0)\right\| e^{-\lambda_{e} t}$ presente no majorante (31), que é obtido utilizando-se a equação do erro (23). Naturalmente, deve-se considerar que o estado $x_{e}(t)$ da equação do erro (23) é modificado no caso em que se elimina o sinal $\pi_{e}(t)$ desta equação.

Observação 2 O uso das transformações $T(\varepsilon)$ e $T^{-1}(\varepsilon)$ na obtenção dos majorantes (31) e (32) para $\left\|\tilde{x}_{e}\right\|$ pode gerar resultados muito conservadores, como se pode concluir do fato de que $\left\|T(\varepsilon) T^{-1}(\varepsilon)\right\|=1$, embora o majorante para esta 
norma estabelecido por $\|T(\varepsilon)\|\left\|T^{-1}(\varepsilon)\right\|=1 / \varepsilon^{n^{*}-1}$ tornase exageradamente grande na medida que $\varepsilon \rightarrow+0$, uma vez que $n^{*} \geq 2$. O uso da norma $\left\|T^{-1}(\varepsilon)\right\|$ na função de modulação do controle em (Emelyanov et al., 1992, eq. (18)) pode resultar em peaking, que pode ser observado no controlador proposto loc. cit. segundo (Oh and Khalil, 1997).

\subsection{Fenômeno peaking}

O fenômeno peaking no observador de alto ganho é caracterizado pelo termo

$$
p\left(t, \varepsilon^{-1}\right):=\frac{k_{1}}{\varepsilon^{n^{*}-1}}\left\|\tilde{x}_{e}(0)\right\| e^{-\frac{\lambda_{\alpha}}{\varepsilon} t},
$$

presente nos majorantes do erro de estimação do estado (31) e (32). Por um lado deve-se escolher o parâmetro $\varepsilon$ suficientemente pequeno para reduzir o erro de estimação residual e para tornar mais rápido o transitório do observador. Por outro lado, isto pode resultar no aumento do pico do erro de estimação do estado durante o transitório inicial na proporção de $1 / \varepsilon^{n^{*}-1}$.

Para ilustrar o fenômeno peaking no HGO, considera-se a equação dinâmica

$$
\dot{\tilde{x}}_{e}(t)=\left[\begin{array}{rr}
-\frac{2}{\varepsilon} & 1 \\
-\frac{1}{\varepsilon^{2}} & 0
\end{array}\right] \tilde{x}_{e}(t), \quad \tilde{x}_{e}(0)=\left[\begin{array}{l}
1 \\
0
\end{array}\right], \quad t \geq 0,
$$

que representa o erro de estimação do estado (34) de um HGO de segunda ordem quando $\bar{U}(t) \equiv 0$ e $\pi_{e}(t) \equiv 0$. Assim, o majorante (31) resulta em

$$
\left\|\tilde{x}_{e}(t)\right\| \leq \frac{k_{1}}{\varepsilon^{n^{*}-1}}\left\|\tilde{x}_{e}(0)\right\| e^{-\frac{\lambda_{\alpha}}{\varepsilon} t}, \quad \forall t \geq 0 .
$$

Notando-se que a margem de estabilidade do polinômio $N_{\alpha}(s)=s^{2}+2 s+1$ é $\bar{\lambda}_{\alpha}=1 s^{-1}$, escolheu-se $\lambda_{\alpha}=$ $0,3 s^{-1}<\bar{\lambda}_{\alpha}$ para que o pico do majorante seja pequeno e o seu transitório seja razoavelmente rápido. A constante $k_{1}=1,2$ foi computada para minimizar o valor de pico do majorante (44) para o valor estipulado de $\lambda_{\alpha}$. A Fig. 3 apresenta a norma do erro de estimação do estado durante o transitório de (43). O fenômeno peaking fica evidente quando o parâmetro $\varepsilon$ é reduzido de 1 para 0,1 . Nota-se que os majorantes estabelecidos pela desigualdade (44) são bastante conservadores. No entanto, o majorante reflete o peaking, pois a sua amplitude e a sua taxa de decaimento aumentam na medida em que o parâmetro $\varepsilon$ é reduzido, tal qual ocorre com a norma do estado.

Um conceito importante sobre observadores de alto ganho é o tempo de extinção do pico, que é definido abaixo conforme a Figura 4.

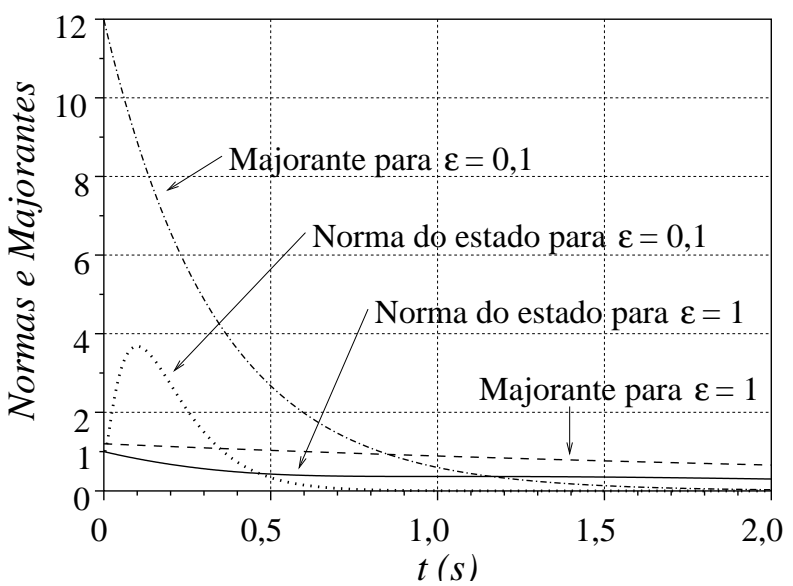

Figura 3: Fenômeno peaking no observador de alto ganho. A norma do erro de estimação do estado $\left\|\tilde{x}_{e}(t)\right\|$ (43) é comparada com o seu majorante (44) para dois valores de $\varepsilon$.

Definição 1 Considere-se a equação da dinâmica do erro de estimação do estado $\left(\tilde{x}_{e}(t)\right)$ de um HGO com $\bar{U}(t) \equiv 0$ e $\pi_{e}(t) \equiv 0$ dada por

$$
\dot{\tilde{x}}_{e}(t)=A_{e}\left(\varepsilon^{-1}\right) \tilde{x}_{e}(t), \quad t \geq 0,
$$

onde a matriz Hurwitz $A_{e}\left(\varepsilon^{-1}\right)$ é definida em (35). O tempo de extinção do pico $\left(t_{e}\right)$ do HGO é o menor valor que satisfaz a desigualdade

$$
\left\|\tilde{x}_{e}(t)\right\| \leq\left\|\tilde{x}_{e}(0)\right\|, \quad \forall t \geq t_{e} \geq 0, \quad \forall \tilde{x}_{e}(0),
$$

para um valor fixo do parâmetro $\varepsilon \in(0,1]$.

O tempo de extinção do pico de um HGO pode ser difícil de ser calculado. Por isso, pode ser mais conveniente estimá-lo através do majorante (31) com $\bar{U}(t) \equiv 0$ e $\pi_{e}(t) \equiv 0$, o que resulta na desigualdade

$$
\frac{k_{1}}{\varepsilon^{n^{*}-1}} e^{-\frac{\lambda_{\alpha}}{\varepsilon} t} \leq 1, \quad \forall t \geq \bar{t}_{e} \geq 0,
$$

onde $\bar{t}_{e} \geq t_{e}$, (vide Figura 4), da qual se conclui que

$$
\bar{t}_{e}=\frac{n^{*}-1}{\lambda_{\alpha}} \varepsilon\left[\left(n^{*}-1\right)^{-1} \ln \left(k_{1}\right)-\ln (\varepsilon)\right] .
$$

Para se compreender a dependência do tempo de extinção do pico em relação ao parâmetro $\varepsilon$, define-se o majorante normalizado do tempo de extinção do pico

$$
\bar{T}_{e}:=\frac{\lambda_{\alpha}}{n^{*}-1} \bar{t}_{e},
$$




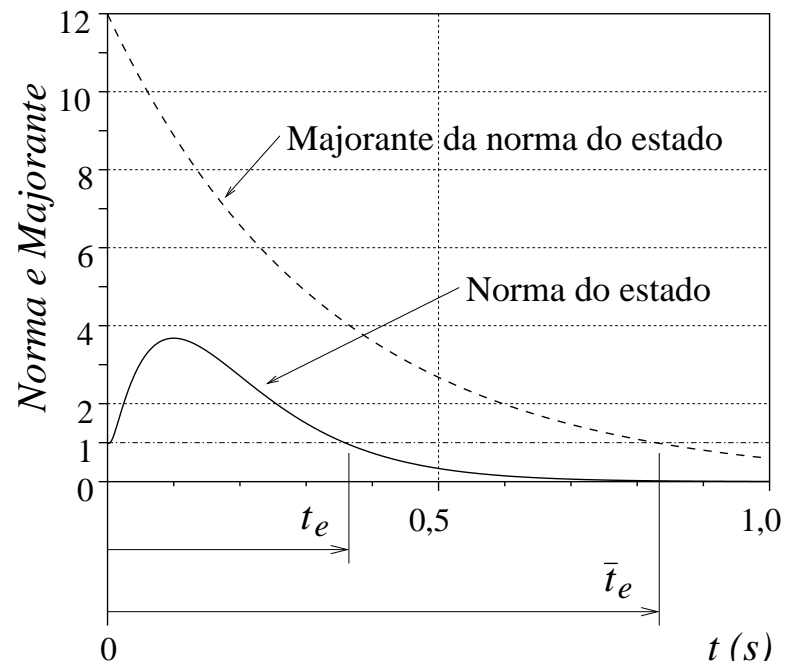

Figura 4: Tempo de extinção do pico $\left(t_{e}\right)$ definido a partir da norma do estado $\left(\left\|\tilde{x}_{e}(t)\right\|\right)$ e o majorante do tempo de extinção do pico $\left(\bar{t}_{e}\right)$ definido a partir do majorante (44). Aqui se utiliza como exemplo o observador de alto ganho com equação do erro de estimação (43) para $\varepsilon=0,1$.

que é dado por

$$
\bar{T}_{e}\left(\varepsilon, K_{p m}\right)=\varepsilon\left[K_{p m}-\ln (\varepsilon)\right],
$$

onde $K_{p m}:=\left(n^{*}-1\right)^{-1} \ln \left(k_{1}\right)$. A família de curvas na Figura 5 representa $\bar{T}_{e}\left(\varepsilon, K_{p m}\right)$ para alguns valores da constante $K_{p m}$. Pode-se concluir que o majorante do tempo de extinção do pico é uniformemente limitado em relação ao parâmetro $\varepsilon \in(0,1]$ e tende a zero na medida que $\varepsilon \rightarrow+0$, para valores fixos dos parâmetros $k_{1} \geq 1, \lambda_{\alpha}>0$ e $n^{*} \geq 2$.

Observação 3 O observador de alto ganho sem peaking proposto por (Chitour, 2002) para sistemas sem sinal de entrada pode ser representado por

$$
\dot{\hat{x}}_{e}=A_{M} \hat{x}_{e}-\left[H\left(t, \varepsilon^{-1}\right)-a_{M}\right] \tilde{e}, \quad \tilde{e}=C_{M} \hat{x}_{e}-e,
$$

onde $H: \mathbb{R}^{+} \times \mathbb{R}^{+} \rightarrow \mathbb{R}^{n^{*}}$ é um vetor variante no tempo. Inicialmente o vetor $H\left(t, \varepsilon^{-1}\right)$ tem norma pequena para que o peaking seja evitado. Então, o vetor $H\left(t, \varepsilon^{-1}\right)$ converge exponencialmente para o vetor $\alpha\left(\varepsilon^{-1}\right)$ a fim de que o erro de estimação seja pequeno após o transitório inicial. No entanto, (Chitour, 2002) reconhece que o observador (51) não teria sucesso em situações reais nas quais perturbações poderiam causar o peaking quando a norma do vetor $H\left(t, \varepsilon^{-1}\right)$ for grande, embora o seu observador elimine o peaking em condições ideais durante o transitório inicial.

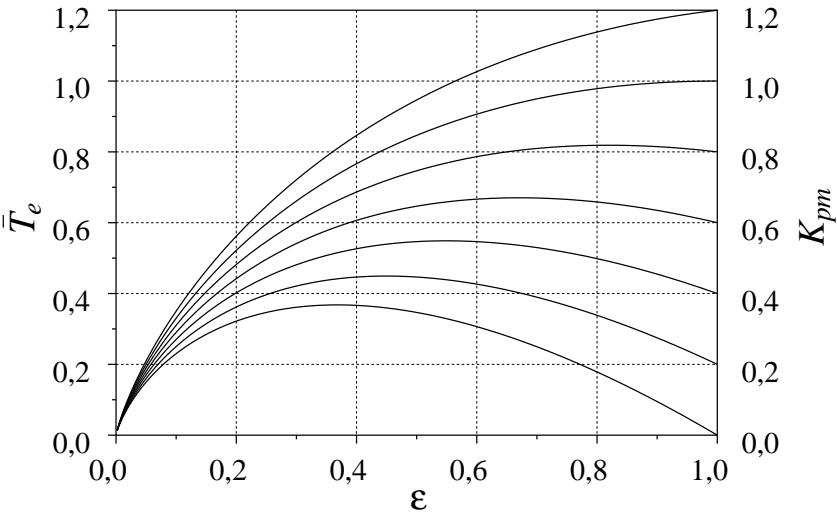

Figura 5: Família de curvas do majorante normalizado do tempo de extinção do pico $\left(\bar{T}_{e}\left(\varepsilon, K_{p m}\right)\right)$.

\section{CONTROLE SEM PEAKING NA PLANTA}

O primeiro controlador proposto utiliza a lei de controle a estrutura variável (25) conjugada com o HGO (26)-(27). Para se evitar o peaking no sinal de controle $u$ e nos sinais da planta, utiliza-se os filtros de estado (11) na síntese da função de modulação $\rho$. No entanto, ocorre o peaking no estado estimado, pois este fenômeno é intrínseco no HGO (26)-(27).

Para analisar a estabilidade do sistema de controle para plantas com grau relativo $n^{*} \geq 2$, levando-se em conta de forma completa as condições iniciais, utiliza-se o vetor de estado $z:=\left[X_{e}^{T}, \tilde{x}_{e}^{T}\right]^{T}$. O Teorema 3 estabelece as propriedades de estabilidade do sistema com todas as equações dos sinais de erro dadas por (14)-(15) e (34). Para $n^{*}=1$ a estabilidade global exponencial é estabelecida no Teorema 1 . No que segue, todos os $k$ 's e $\lambda$ 's simbolizam constantes positivas genéricas, $\mathcal{K}$ 's são funções da classe $\mathcal{K}$ e a norma de um operador $(\|\cdot\|)$ é induzida pela norma $\mathcal{L}_{\infty e}$.

Teorema 3 Para $N=n^{*}-1 \geq 1$, considere-se a planta (2), a lei de controle (25) e (18), os filtros de estado (11) e o observador (26)-(27). Se as hipóteses (A1)-(A6) forem satisfeitas e a função de modulação $\rho$ satisfizer a desigualdade (19) e a hipótese (A7), então, para $\varepsilon>0$ suficientemente pequeno, o sistema composto pelas equações dos erros (14)-(15) e (34) com estado z será globalmente exponencialmente estável em relação a um conjunto residual que converge exponencialmente para ordem $\varepsilon$, isto é, existem constantes $k_{z}, \lambda_{z}>0 e$ uma função $\mathcal{K}_{X}(\varepsilon)$ da classe $\mathcal{K}$ tais que, $\forall z(0), \forall t \geq 0$,

$$
\begin{aligned}
& \|z(t)\| \leq\left[\frac{k_{z}}{\varepsilon^{n^{*}-1}}\|z(0)\|+\mathcal{K}_{X}(\varepsilon)\right] e^{-\lambda_{z} t}+O(\varepsilon) \\
& |e(t)| \mathrm{e}\left\|X_{e}(t)\right\| \leq\left[k_{z}\|z(0)\|+\mathcal{K}_{X}(\varepsilon)\right] e^{-\lambda_{z} t}+O(\varepsilon)
\end{aligned}
$$


Prova: Vide Apêndice B.

Observação $4 \mathrm{O}$ controlador de rastreamento de trajetória de (Oh and Khalil, 1997) é baseado em estrutura variável e num HGO. Esse controlador evita o fenômeno peaking na planta através da saturação global do sinal de controle. No entanto, os resultados obtidos loc. cit. garantem apenas a estabilidade semi-global e o estado da equação do erro de rastreamento converge em tempo finito para um conjunto residual de ordem $\sqrt{\varepsilon}$. Nesses aspectos, esses resultados não são tão bons quanto estes estabelecidos no Teorema 3 , em que se obtém a estabilidade global e os estados das equações dos erros convergem exponencialmente para um conjunto residual de ordem $\varepsilon$. Em contrapartida, aqui a planta deve ser linear e invariante no tempo, ao passo que a abordagem de (Oh and Khalil, 1997) foi orientada a plantas não-lineares.

\section{CONTROLADOR LIVRE DE PEAKING}

O segundo controlador aqui proposto utiliza um $\mathrm{HGO}$ para o estado da equação do erro (23) escalonado pela transformação

$$
\zeta=T(\varepsilon) x_{e}, T(\varepsilon)=\operatorname{diag}\left\{1, \varepsilon, \ldots, \varepsilon^{n^{*}-1}\right\} .
$$

O HGO livre do peaking no estado estimado é obtido através da aplicação da transformação $\hat{\zeta}=T(\varepsilon) \hat{x}_{e}$ ao HGO (26)(27), resultando no observador dinâmico

$$
\begin{aligned}
\varepsilon \dot{\hat{\zeta}} & =\bar{A}_{M}(\varepsilon) \hat{\zeta}+\varepsilon^{n^{*}} B_{M} K^{\text {nom }} U-\left[\bar{\alpha}-\bar{a}_{M}(\varepsilon)\right] \tilde{e}, \\
\tilde{e} & =C_{M} \hat{\zeta}-e,
\end{aligned}
$$

onde $\bar{A}_{M}(\varepsilon)=\varepsilon T(\varepsilon) A_{M} T^{-1}(\varepsilon), \bar{\alpha}=\varepsilon T(\varepsilon) \alpha\left(\varepsilon^{-1}\right) \mathrm{e}$ $\bar{a}_{M}(\varepsilon)=\varepsilon T(\varepsilon) a_{M}$.

Conclui-se que este observador é livre do peaking pois a equação da dinâmica do erro de estimação do estado $\bar{x}_{e}(t):=$ $\hat{\zeta}(t)-\zeta(t)$ é dada por (38), que não exibe peaking conforme o majorante para norma do erro de estimação do estado (39).

A lei de controle a estrutura variável neste caso é definida pela superfície de chaveamento $\sigma\left(\hat{x}_{e}\right)=0$, onde $\sigma\left(\hat{x}_{e}\right):=$ $S \hat{x}_{e}=S T^{-1}(\varepsilon) \hat{\zeta}$. Uma vez que o sinal $\sigma\left(T^{-1}(\varepsilon) \hat{\zeta}\right)$ poderia apresentar peaking devido ao uso da transformação $T^{-1}(\varepsilon)$ cuja norma é $\varepsilon^{1-n^{*}}$, este é escalado adequadamente resultando no sinal $\bar{\sigma}(\hat{\zeta}):=\varepsilon^{n^{*}-1} S T^{-1}(\varepsilon) \hat{\zeta}$ que não exibe peaking. Assim a lei de controle é dada por

$$
u=u^{\mathrm{nom}}+U, \quad U=-\rho \operatorname{sgn}(\bar{S}(\varepsilon) \hat{\zeta}),
$$

onde $\bar{S}(\varepsilon):=\varepsilon^{n^{*}-1} S T^{-1}(\varepsilon)$ e o controle nominal $u^{\text {nom }}$ é dado por (18). Este controlador é descrito na Tabela $1 \mathrm{e}$ no diagrama de blocos na Fig. 6. Neste diagrama de blocos foi assinalado o laço do modo deslizante "ideal", no qual a função de transferência do sinal $U$ para o sinal $\bar{\sigma}$ é de fase mínima e possui grau relativo unitário, o que possibilita a ocorrência do modo deslizante (Bondarev et al., 1985). O relé que gera o controle chaveado faz parte de uma malha de realimentação que passa diretamente através do observador, que realiza o bypass da dinâmica da planta. Assim, o chattering que poderia ser causado por dinâmicas parasitas na planta, sensores e atuadores pode ser evitado pelo uso do observador na malha com controle por modo deslizante (Young et al., 1999), (Utkin et al., 1999, Seção 8.3).

Tabela 1: Algoritmo proposto para o controle por modelo de referência de sistemas lineares com grau relativo $n^{*}>1$ livre

\begin{tabular}{|c|c|}
\hline Modelo de referência & $\begin{array}{l}\dot{x}_{M}=A_{M} x_{M}+B_{M} r, \\
y_{M}=C_{M} x_{M} .\end{array}$ \\
\hline Erro de saída & $e(t)=y(t)-y_{M}(t)$. \\
\hline Filtros de estado & $\omega_{1}=\frac{A(s)}{\Lambda(s)} u, \quad \omega_{2}=\frac{A(s)}{\Lambda(s)} y$ \\
\hline Vetor regressor & 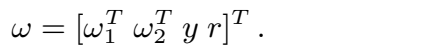 \\
\hline Lei de controle & $\begin{array}{l}u=u^{\mathrm{nom}}+U, \\
U=-\rho \operatorname{sgn}(\bar{\sigma}) \\
\bar{\sigma}=\bar{S}(\varepsilon) \hat{\zeta} \\
\bar{S}(\varepsilon)=\varepsilon^{n^{*}-1} S T^{-1}(\varepsilon) .\end{array}$ \\
\hline Controle nominal & $u^{\mathrm{nom}}=\left(\theta^{\mathrm{nom}}\right)^{T} \omega$ \\
\hline Função de modulação & $\begin{array}{l}\rho=\delta+c_{1}\|\omega\|+\hat{d}(t) \\
\hat{d}(t)=\bar{d}(t)+\frac{c_{2}}{s+\gamma_{d}} * \bar{d}(t)\end{array}$ \\
\hline Observador de alto ganho & $\begin{aligned} \varepsilon \dot{\hat{\zeta}}= & \bar{A}_{M}(\varepsilon) \hat{\zeta}+\varepsilon^{n^{*}} B_{M} \frac{K_{p}^{\text {nom }}}{K_{M}} U \\
& -\left[\bar{\alpha}-\bar{a}_{M}(\varepsilon)\right] \tilde{e}, \\
\tilde{e}= & C_{M} \hat{\zeta}-e .\end{aligned}$ \\
\hline
\end{tabular}
de peaking. A função de modulação apresentada nesta tabela pode ser substituída por qualquer outra função de modulação que satisfaça a desigualdade (19) e a hipótese (A7).

Para analisar a estabilidade do sistema de controle que utiliza o HGO livre do peaking, utiliza-se o vetor de estado $\bar{z}:=\left[X_{e}^{T}, \bar{x}_{e}^{T}\right]^{T}$. O Teorema 4 estabelece as propriedades de estabilidade do sistema com todas as equações dos sinais de erro (14)-(15) e (38).

Teorema 4 Para $N=n^{*}-1 \geq 1$, considere-se a planta (2), a lei de controle (56) e (18), os filtros de estado (11) e o observador (55). Se as hipóteses (A1)-(A6) forem satisfeitas e a função de modulação $\rho$ satisfizer a desigualdade (19) e a hipótese (A7), então, para $\varepsilon>0$ suficientemente pequeno, $o$ 


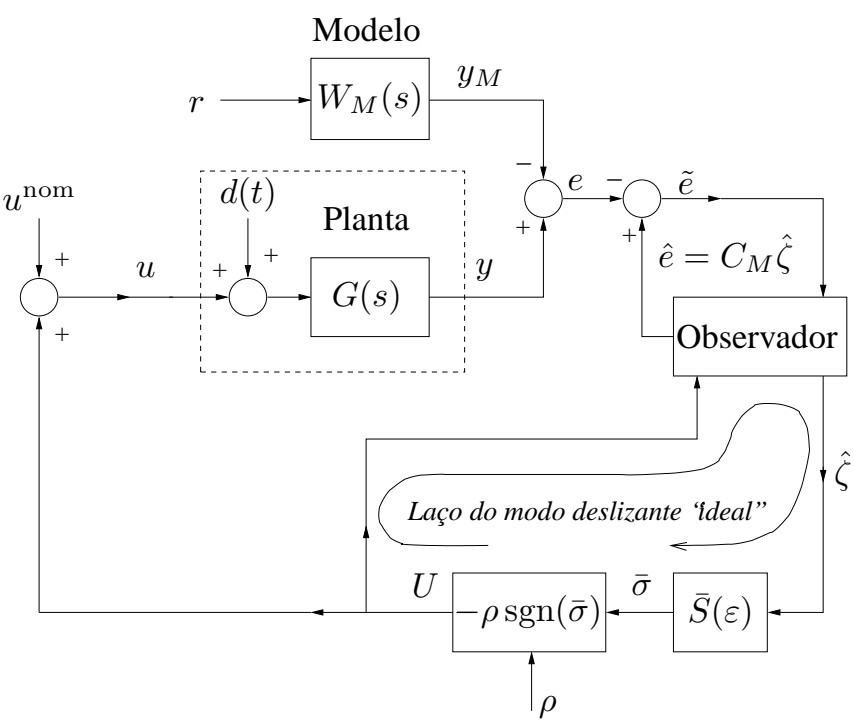

Figura 6: Controlador por modelo de referência de sistemas lineares com grau relativo $n^{*} \geq 2$ livre de peaking. Os filtros de estado e a síntese do sinal $\rho$ foram omitidos para simplificar o diagrama.

sistema composto pelas equações dos erros (14)-(15) e (38) com estado $\bar{z}$ será globalmente exponencialmente estável em relação a um conjunto residual que converge exponencialmente para ordem $\varepsilon$, isto é, existem constantes $k_{z}, \lambda_{z}>0 e$ uma função $\mathcal{K}_{X}(\varepsilon)$ da classe $\mathcal{K}$ tais que, $\forall \bar{z}(0), \forall t \geq 0$,

$$
|e(t)| \text { e }\|\bar{z}(t)\| \leq\left[k_{z}\|\bar{z}(0)\|+\mathcal{K}_{X}(\varepsilon)\right] e^{-\lambda_{z} t}+O(\varepsilon) .
$$

Prova: Segue a prova do Teorema 3, considerando-se que o erro de estimação é dado por $\bar{x}_{e}(t)=T(\varepsilon) \tilde{x}_{e}(t)$.

Observação 5 Os Teoremas 3 e 4 estabelecem a estabilidade global exponencial dos controladores propostos baseados em estrutura variável e em HGOs em relação a um conjunto residual. Os sinais $r(t)$ e $d(t)$ que excitam o sistema resultam no termo exponencialmente decrescente $\mathcal{K}_{X}(\varepsilon) e^{-\lambda_{z} t}$ presente nos majorantes do estado (52)-(53) e (57). No caso autônomo, i.e., $r(t) \equiv 0$ e $d(t) \equiv 0$, pode-se escolher $\mathcal{K}_{X}(\varepsilon) \equiv 0$. Em contraste, o VS-MRAC para sistemas SISO (Hsu et al., 1997) é globalmente exponencialmente estável em relação a um conjunto residual de ordem $\tau$ sem o termo $\mathcal{K}_{X}(\tau) e^{-a t}$ nos majorantes das normas dos erros, ainda que as funções $r(t)$ e $d(t)$ estejam ativas.

\section{EXEMPLO DE APLICAÇÃO}

Para ilustrar a aplicação do controlador proposto, escolheuse um servomecanismo em que se controla a posição de um carro acionado por um motor DC com ímãs permanentes ao longo de um trilho (Apkarian, 1995, Seções 3.1 e 5.2.1). A simplicidade deste servomecanismo clássico facilita o desenvolvimento do exemplo do projeto do controlador na $\mathrm{Se}$ ção 10.2. Além disso, os experimentos descritos na Seção 10.4 permitem a avaliação do algoritmo proposto em condições realistas com perturbações (e.g., atrito seco), ruído de medição, dinâmica não modelada e grandes incertezas paramétricas.

A posição do carro $(y)$ é medida por um potenciômetro multivoltas que gera uma tensão $\left(e_{p}\right)$ proporcional à posição. $\mathrm{O}$ eixo do motor e o eixo do potenciômetro são conectados por engrenagens a uma cremalheira instalada no trilho, conforme a Figura 7.

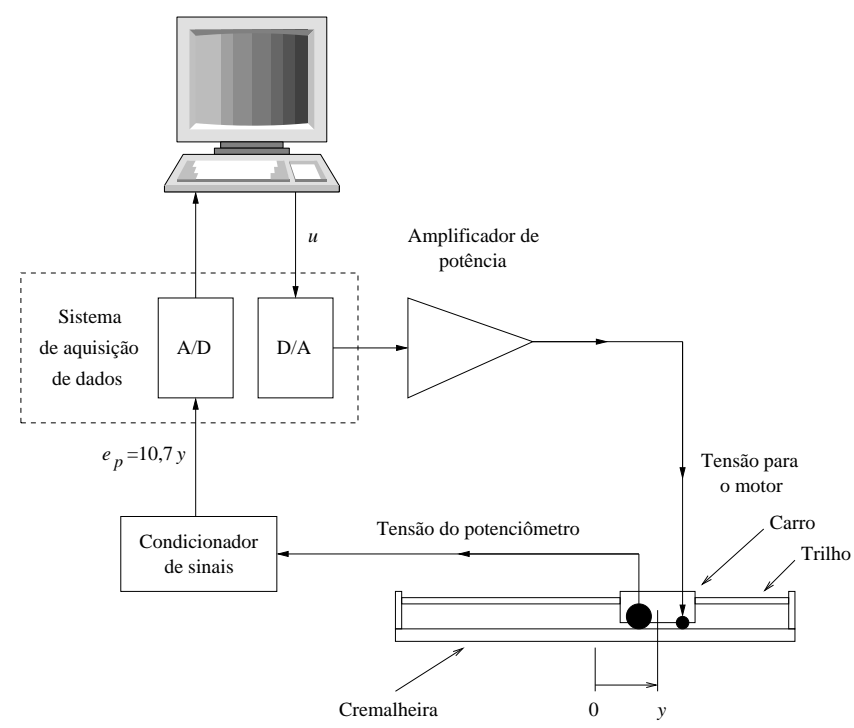

Figura 7: Diagrama do servomecanismo.

A interface com o computador utiliza um conversor A/D e um conversor D/A com resolução de 12 bits e tensões de entrada e de saída na faixa de $-5 \mathrm{~V}$ a $+5 \mathrm{~V}$. O sinal de controle (u) atua na tensão enviada ao motor do carro.

$\mathrm{O}$ algoritmo de controle baseado no HGO livre de peaking (Tabela 1) é codificado num computador digital utilizandose integração numérica pelo método de Euler. As variáveis são amostradas com periodicidade de $1 \mathrm{~ms}$.

\subsection{Modelo da dinâmica do carro}

A modelagem da dinâmica do carro permite concluir que a sua função de transferência é dada por

$$
G(s) \approx \frac{K_{p}}{s\left(s+p_{1}\right)}
$$


onde os parâmetros incertos pertencem às faixas: $p_{1} \in$ $[-15 ;-9,5]$ e $K_{p} \in[1,59 ; 2,99]$. Para o projeto do controle nominal, estimou-se a função de transferência da planta nominal $\left(G^{\text {nom }}(s)=2,41 /[s(s+13,4)]\right)$ a partir da sua resposta em frequiência medida experimentalmente. A perturbação mais significativa é o atrito, que é incorporado como perturbação de entrada (tensão). Essa perturbação é uniformemente limitada por $\bar{d}_{\text {sup }}=0,3 \mathrm{~V}$, valor obtido experimentalmente.

\subsection{Projeto}

O modelo de referência escolhido é $W_{M}(s)=50 /[(s+5)(s+$ $10)]$, que tem ganho DC unitário. Escolheu-se o polinômio $L(s)=s+10$ para cancelar o pólo em $-10 s^{-1}$ do modelo de referência. Os filtros de estado são escolhidos $\operatorname{com} \lambda(s)=$ $s+10$. O vetor de parâmetros utilizado no controle nominal é calculado para casar a planta nominal $G^{\mathrm{nom}}(s)$ com o modelo de referência, o que resulta na solução da equação Diofantina (13) dada por $\theta^{\text {nom }}=\left[\begin{array}{llll}-1,6 & -22,6 & -18,5 & 20,7\end{array}\right]^{T}$.

A função de modulação escolhida $\left(\rho(t)=c_{1}\|\omega(t)\|+\hat{d}(t)\right)$ deve ser projetada de forma que o majorante (19) seja satisfeito considerando-se que os parâmetros da planta são incertos. Além disso, deve-se manter o sinal de controle com pequena amplitude, o que pode ser conseguido se as constantes da função de modulação forem pequenas. A constante $c_{1}=44$ da função de modulação foi calculada numericamente de forma que a desigualdade

$$
c_{1} \geq \max _{\substack{K_{p} \in[1,59 ; 2,99] \\ p_{1} \in[-15,0 ;-9,5]}}\left\|\theta^{*}\left(K_{p}, p_{1}\right)-\theta^{\text {nom }}\right\|,
$$

seja satisfeita, onde $\theta^{*}\left(K_{p}, p_{1}\right)$ é a solução da equação Diofantina (13) que depende dos parâmetros incertos da planta.

Uma vez que o atrito é uniformemente limitado, utilizou-se o majorante $\hat{d}(t) \equiv 0,47$ para permitir o cancelamento do efeito do atrito. Esse é um majorante para o valor em regime permanente da resposta do filtro (8) a um degrau de amplitude $\bar{d}_{\text {sup }}$, dado por

$$
\hat{d} \geq\left(\max _{\substack{K_{p} \in[1,59 ; 2,99] \\ p_{1} \in[-15,0 ;-9,5]}}\left|1-\theta_{1}^{* T}\left(K_{p}, p_{1}\right) \frac{A(0)}{\Lambda(0)}\right|\right) \bar{d}_{\mathrm{sup}} .
$$

A computação de cada constante foi tratada como um problema de otimização. Naturalmente, o método de otimização deve ser adequado ao sistema e às suas incertezas.

\subsection{Resultados de simulação}

Nas simulações e nos experimentos (Seção 10.4) o sinal de referência é uma onda quadrada com amplitude $20 \mathrm{~mm}$ e frequiência $3 \mathrm{rad} / \mathrm{s}$. Nas simulações não há perturbação aplicada na entrada da planta, embora no sistema real haja atrito seco que poderia ser incluído como perturbação de entrada. No entanto, para se avaliar os efeitos das incertezas paramétricas, nas simulações os valores dos parâmetros da função de transferência do servomecanismo são bastante distintos dos valores nominais, isto é, $G^{\text {dist }}(s)=1,59 /[s(s+15)]$. Todas as condições iniciais são nulas, exceto a posição inicial do carro que é $y(0)=100 \mathrm{~mm}$.

As Figuras 8 e 9 apresentam os resultados de simulação obtidos para dois valores do parâmetro $\varepsilon$ a fim de se avaliar a influência de $\varepsilon$ no desempenho do sistema de controle. $O$ bom desempenho do controlador é evidenciado pela rápida convergência do erro de saída que pode ser apreciada na Figura 8. Conforme esperado, o erro residual após o transitório inicial é significativamente reduzido na medida em que $\varepsilon$ passa de 0,1 (Figura 8.a) para 0,01 (Figura 8.b).

O fenômeno peaking não ocorre em nenhum dos sinais simulados, uma vez que este controlador é livre de peaking. Particularmente, nota-se que o peaking não ocorre nas variáveis de estado estimadas $\hat{\zeta}_{1}$ e $\hat{\zeta}_{2}$, pois quando o valor do parâmetro $\varepsilon$ é reduzido de 0,1 para 0,01 as amplitudes dos picos destas variáveis ficam praticamente inalteradas, bem como as constantes de tempo dos modos mais lentos, conforme se percebe comparando-se a Figura 9.a com a Figura 9.b.

\subsection{Resultados experimentais}

Para a verificação da importância do controle a estrutura variável, na Fig. 10 aplica-se apenas o controle linear nominal (i.e., $\left.u=\left(\theta^{\text {nom }}\right)^{T} \omega\right)$ e a massa do carro é nominal $(0,455 \mathrm{~kg})$. Verifica-se que esse controle é incapaz de realizar o rastreamento preciso. A principal causa dos erros exagerados é o atrito seco, cujo efeito é mais evidente quando a velocidade do carro é pequena.

Na Fig. 11, o controle a estrutura variável (25) inclui o controle nominal, mas a massa do carro é aumentada para $0,665 \mathrm{~kg}$. Esta alteração é bastante significativa em comparação com a massa nominal (46\%). Obtém-se um rastreamento bastante preciso, ainda que a massa do carro tenha sido aumentada.

Uma característica importante é a natureza do sinal de controle $u$. Na Fig. 12 o sinal de controle linear nominal é bastante suave e de pequena amplitude. O algoritmo baseado em estrutura variável e no HGO sem peaking resulta no sinal controle chaveado em alta freqüência e de grande amplitude apresentado na Fig. 13. Conseqüentemente, a potência dissipada em calor no motor foi maior no esquema baseado em estrutura variável do que no caso do controlador linear nominal. Em ambos experimentos a massa do carro é nominal. 


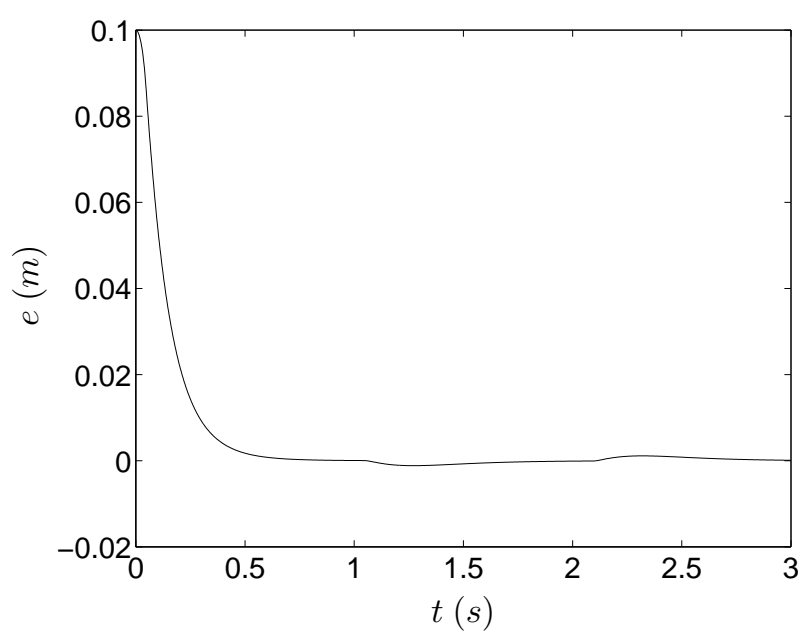

(a)

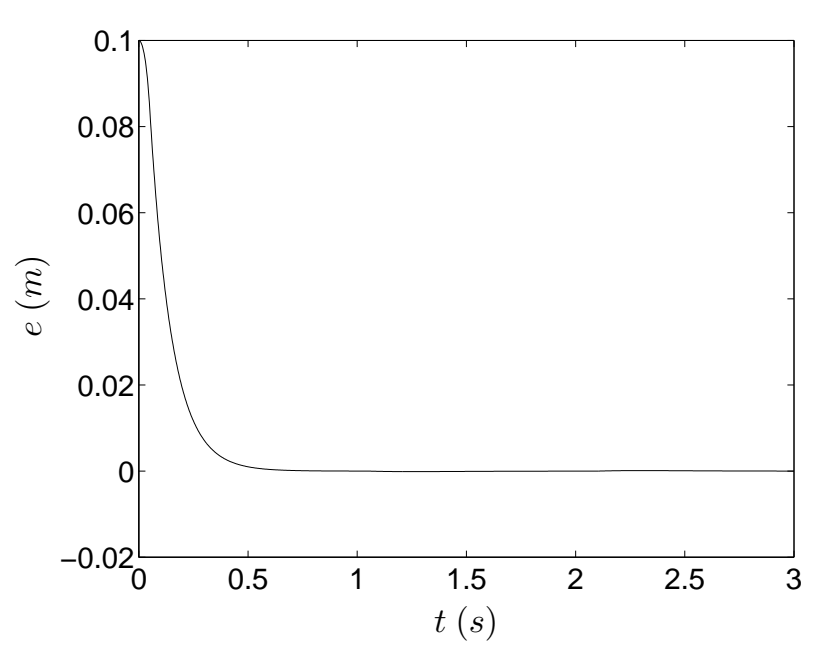

(b)

Figura 8: Simulações do erro de saída para: (a) $\varepsilon=0,1 \mathrm{e}$ (b) $\varepsilon=0,01$. O sinal de referência é uma onda quadrada.

Na Fig. 13 observa-se que inicialmente o sinal $u$ ultrapassa um pouco as tensões de saturação do conversor D/A $( \pm 5 \mathrm{~V})$, o que não prejudicou o desempenho do controlador.

Comprovou-se que o uso do observador reduziu as oscilações em baixa freqüência (chattering), que poderiam ser causadas pela dinâmica não modelada (e.g., dinâmica elétrica da armadura do motor, filtro antialiasing no sistema de aquisição de dados). Apesar disto, o controle a estrutura variável resulta em vibrações que poderiam desgastar os componentes mecânicos. As causas aparentes dessas vibrações são o ruído na medição da posição $y$ e a freqüência de amostragem relativamente baixa $(1 \mathrm{kHz})$ que pôde ser obtida com o sistema de aquisição de dados utilizado.

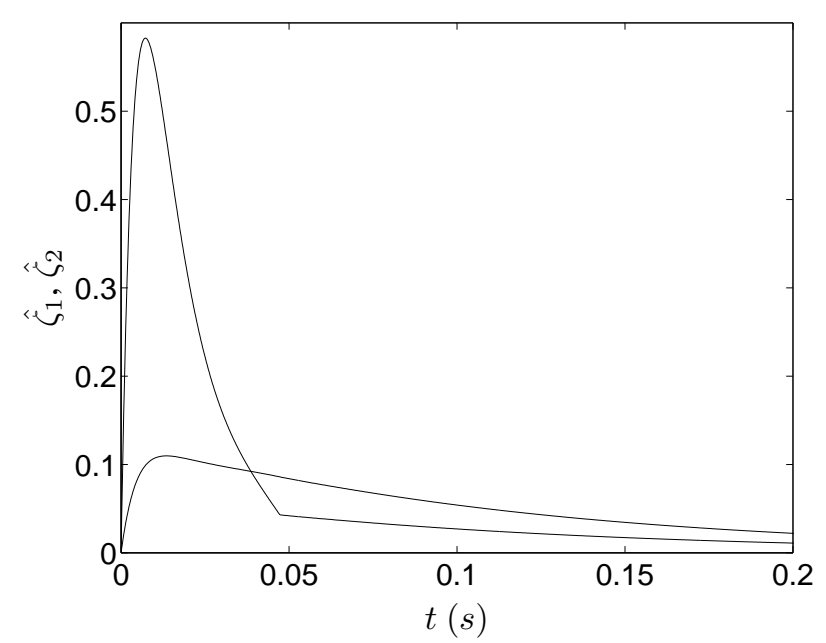

(a)

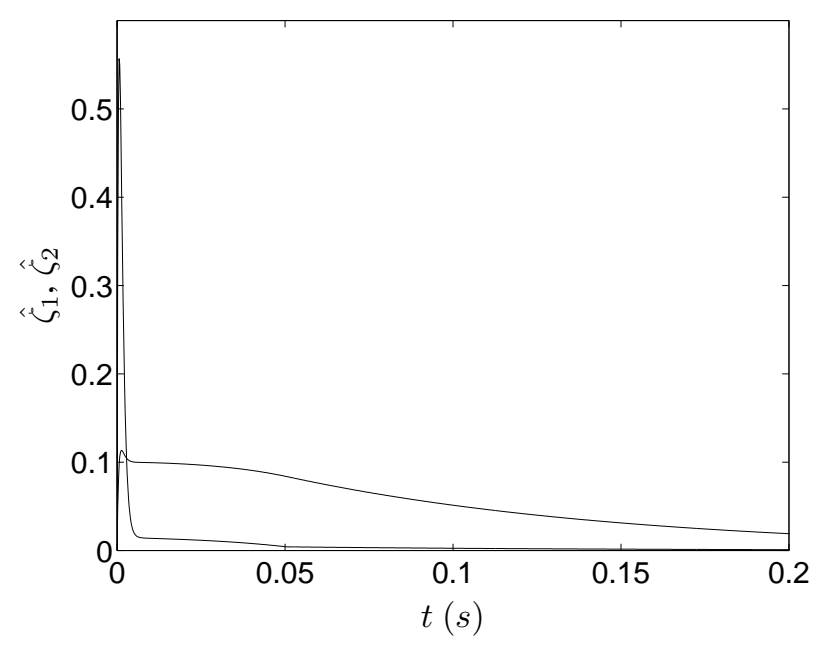

(b)

Figura 9: Simulações das variáveis de estado estimadas pelo observador de alto ganho para: (a) $\varepsilon=0,1$ e (b) $\varepsilon=0,01$.

\section{CONCLUSÃO}

Neste artigo foi desenvolvido um controlador baseado em estrutura variável e observador de alto ganho para sistemas lineares SISO incertos que, em malha fechada, resulta em um sistema globalmente exponencialmente estável em relação a um conjunto residual e livre do fenômeno peaking. A principal contribuição deste artigo é a introdução de um novo mecanismo para a eliminação do fenômeno de peaking. Em (Esfandiari and Khalil, 1992; Emelyanov et al., 1992) o uso do HGO resultou em peaking, conforme foi mostrado em (Oh and Khalil, 1995). Algumas alternativas utilizadas para se evitar o peaking são:

1. A limitação do sinal de controle empregando-se satura- 


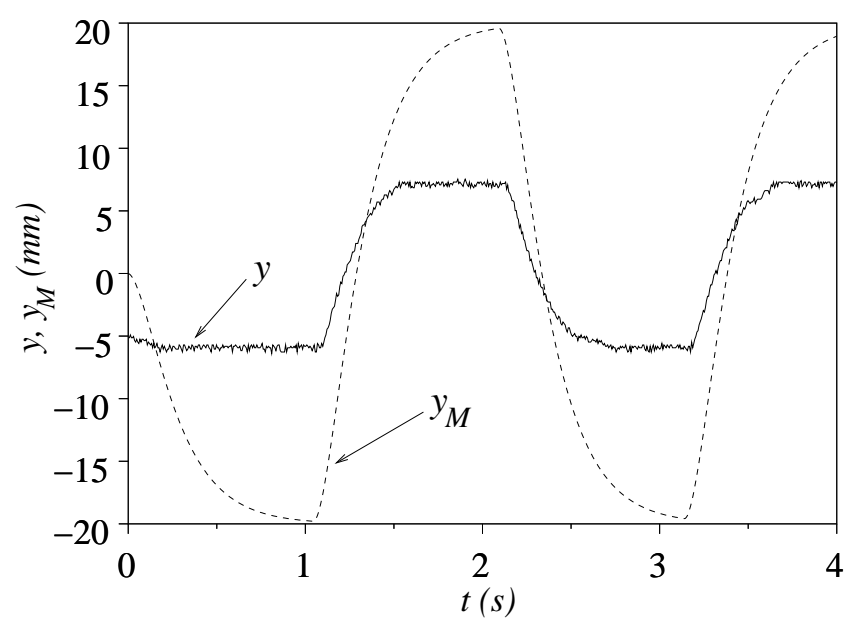

Figura 10: Experimento de rastreamento de trajetória com o controle linear nominal.

ções (Oh and Khalil, 1995; Oh and Khalil, 1997), cuja pena é se obter apenas a estabilidade semi-global.

2. O uso de um HGO com ganho variante no tempo (Chitour, 2002), vide Observação 3.

3. O uso de um observador de semi-alto ganho (Lu and Spurgeon, 1998; Lu and Spurgeon, 1999), que de fato é um HGO em que se usa um valor para o parâmetro $\varepsilon$ que não seja muito conservador, mas que é computado para se garantir a estabilidade do sistema em malha fechada.

Uma característica interessante do esquema de controle aqui proposto é que este guarda alguma semelhança com o VSMRAC para sistemas SISO (Hsu et al., 1994; Hsu et al., 1997) e o UV-MRAC para sistemas MIMO (Hsu et al., 2002), conforme se poderia observar comparando-se o diagrama de blocos do UV-MRAC, que é semelhante ao diagrama de blocos do VS-MRAC, com o diagrama de blocos do controlador baseado no HGO (Fig. 6). O VS-MRAC, o UV-MRAC e o controlador aqui proposto são livres do fenômeno peaking e globalmente exponencialmente estáveis em relação a conjuntos residuais da ordem de pequenos parâmetros, que em condições ideais devem ser diminuídos para se reduzir o erro de rastreamento.

Os resultados experimentais obtidos com o controlador baseado em estrutura variável e no HGO livre de peaking indicam que se pode obter bom desempenho em situações realistas com perturbações não-lineares, ruído de medição, dinâmica não modelada e grandes incertezas paramétricas.

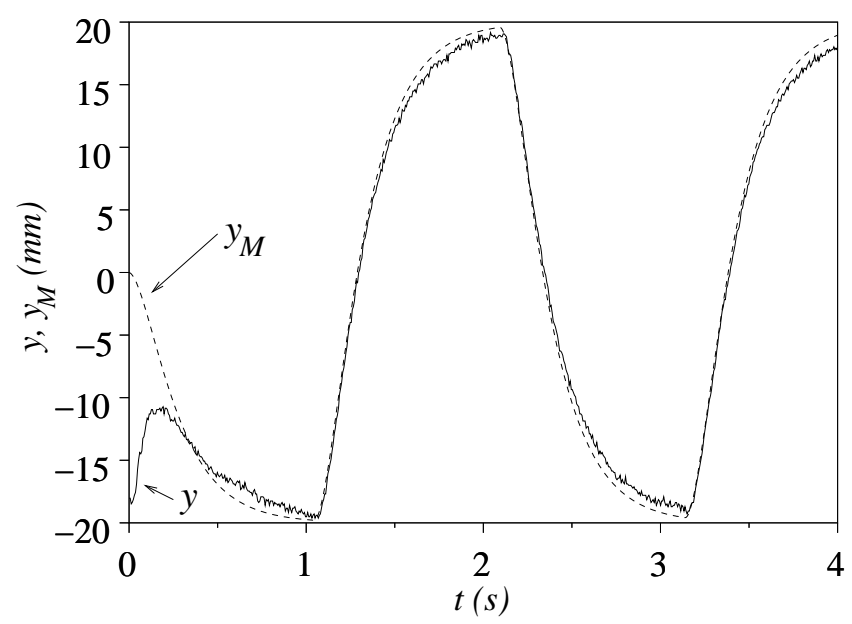

Figura 11: Experimento do rastreamento de trajetória com o controle a estrutura variável.

\section{AGRADECIMENTOS}

Este trabalho foi parcialmente financiado pela FAPERJ e pelo CNPq.

\section{REFERÊNCIAS}

Apkarian, J. (1995). A Comprehensive and Modular Laboratory for Control Systems Design and Implementation, Quanser Consulting.

Bondarev, A. G., Bondarev, S. A., Kostyleva, N. E. and Utkin, V. I. (1985). Sliding modes in systems with asymptotic state observers, Autom. Remote Control 46(6): 679-684. Pt. 1.

Chang, P. H. and Lee, J. W. (1996). A model reference observer for time-delay control and its application to robot trajectory control, IEEE Trans. Contr. Syst. Tech. 4(1): 2-10.

Chien, C.-J., Sun, K.-C., Wu, A.-C. and Fu, L.-C. (1996). A robust MRAC using variable structure design for multivariable plants, Automatica 32(6): 833-848.

Chitour, Y. (2002). Time-varying high-gain observers for numerical differentiation, IEEE Trans. Aut. Contr. 47(9): 1565-1569.

Cunha, J. P. V. S. (2004). Controle de Sistemas Multivariáveis Incertos por Realimentação de Saída e Modo Deslizante, $\mathrm{PhD}$ thesis, Programa de Engenharia Elétrica, COPPE/UFRJ, Rio de Janeiro.

Cunha, J. P. V. S., Costa, R. R. and Hsu, L. (2003). Design of first order approximation filters applied to sli- 


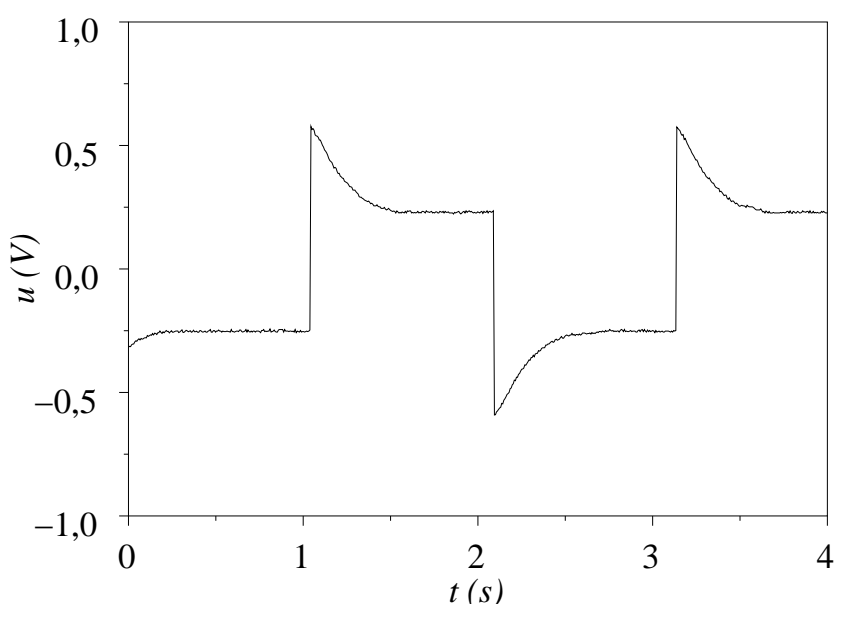

Figura 12: Sinal de controle experimental com o controle nominal.

ding mode control, Proc. IEEE Conf. on Decision and Control, Maui, Hawaii, pp. 3531-3536.

Cunha, J. P. V. S., Hsu, L., Costa, R. R. and Lizarralde, F. (2003). Output-feedback model-reference sliding mode control of uncertain multivariable systems, IEEE Trans. Aut. Contr. 48(12): 2245-2250.

Edwards, C. and Spurgeon, S. K. (1996). Robust output tracking using a sliding-mode controller/observer scheme, Int. J. Contr. 64(5): 967-983.

Edwards, C. and Spurgeon, S. K. (1998). Sliding Mode Control: Theory and Applications, Taylor \& Francis Ltd.

Emelyanov, S. V., Korovin, S. K., Nersisian, A. L. and Nisenzon, Y. Y. (1992). Output feedback stabilization of uncertain plants: a variable structure systems approach, Int. J. Contr. 55(1): 61-81.

Esfandiari, F. and Khalil, H. K. (1992). Output feedback stabilization of fully linearizable systems, Int. J. Contr. 56: 1007-1037.

Hsu, L. (1997). Smooth sliding control of uncertain systems based on a prediction error, Int. J. on Robust and Nonlinear Control 7: 353-372.

Hsu, L., Araújo, A. D. and Costa, R. R. (1994). Analysis and design of I/O based variable structure adaptive control, IEEE Trans. Aut. Contr. 39(1): 4-21.

Hsu, L. and Costa, R. R. (1989). Variable structure model reference adaptive control using only input and output measurement: Part I, Int. J. Contr. 49(2): 399-416.

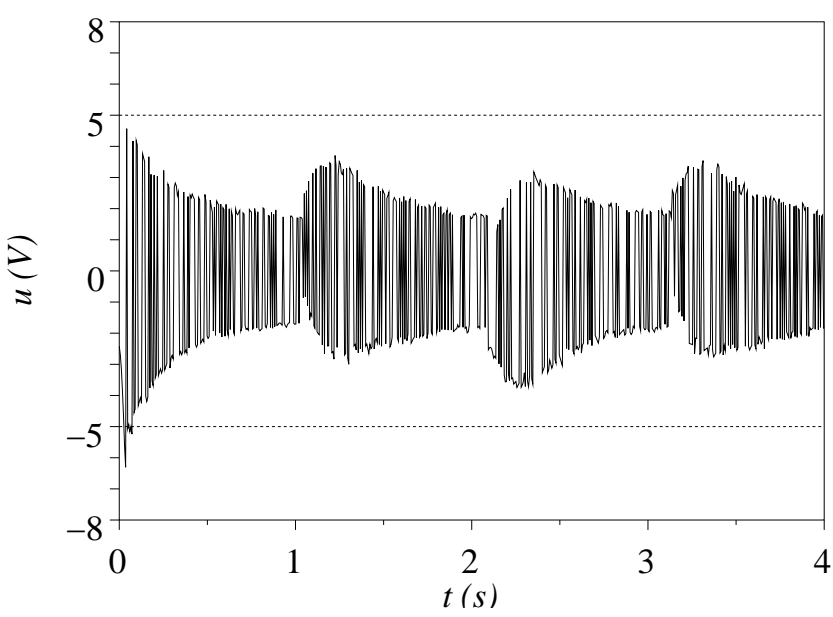

Figura 13: Sinal de controle experimental do algoritmo a estrutura variável incluindo o controle nominal.

Hsu, L., Costa, R. R. and Cunha, J. P. V. S. (2003). Modelreference output-feedback sliding mode controller for a class of multivariable nonlinear systems, Asian Journal of Control 5(4): 543-556.

Hsu, L., Cunha, J. P. V. S., Costa, R. R. and Lizarralde, F. (2002). Multivariable output-feedback sliding mode control, in X. Yu and J.-X. Xu (eds), Variable Structure Systems: Towards the 21st Century, Springer-Verlag, pp. 283-313.

Hsu, L., Lizarralde, F. and Araújo, A. D. (1997). New results on output-feedback variable structure adaptive control: design and stability analysis, IEEE Trans. Aut. Contr. 42(3): 386-393.

Ioannou, P. A. and Sun, J. (1996). Robust Adaptive Control, Prentice-Hall.

Kailath, T. (1980). Linear Systems, Prentice Hall.

Lu, X.-Y. and Spurgeon, S. K. (1998). Output feedback stabilization of SISO nonlinear systems via dynamic sliding modes, Int. J. Contr. 70(5): 735-759.

Lu, X.-Y. and Spurgeon, S. K. (1999). Output feedback stabilization of MIMO non-linear systems via dynamic sliding mode, Int. J. of Robust and Nonlinear Control 9: 275-305.

Min, L. J. and Hsu, L. (2000). Sliding controller for output feedback of uncertain nonlinear systems: global and semi-global results, Proc. 6th IEEE Int. Workshop on Variable Structure Sys., Gold Coast, Australia, pp. 169178. 
Oh, S. and Khalil, H. K. (1995). Output feedback stabilization using variable structure control, Int. J. Contr. 62(4): 831-848.

Oh, S. and Khalil, H. K. (1997). Nonlinear output-feedback tracking using high-gain observer and variable structure control, Automatica 33(10): 1845-1856.

Sastry, S. and Bodson, M. (1989). Adaptive Control: Stability, Convergence and Robustness, Prentice-Hall.

Sussmann, H. J. and Kokotović, P. V. (1991). The peaking phenomenon and the global stabilization of nonlinear systems, IEEE Trans. Aut. Contr. 36(4): 424-440.

Tao, G. and Ioannou, P. A. (1989). A MRAC for multivariable plants with zero residual tracking error, Proc. IEEE Conf. on Decision and Control, Tampa, USA, pp. 1597-1600.

Utkin, V., Guldner, J. and Shi, J. (1999). Sliding Mode Control in Electromechanical Systems, Taylor \& Francis Ltd.

Utkin, V. I. (1978). Sliding Modes and Their Application in Variable Structure Systems, MIR.

Walcott, B. L. and Żak, S. (1988). Combined observercontroller synthesis for uncertain dynamical systems with applications, IEEE Trans. Syst. Man and Cyber. 18(1): 88-104.

Young, K. D., Utkin, V. I. and Özgüner, U. (1999). A control engineer's guide to sliding mode control, IEEE Trans. Contr. Syst. Tech. 7(3): 328-342.

\section{A LEMAS}

Neste Apêndice são apresentados lemas que são aplicados no desenvolvimento do VS-MRAC. O Lema 5 é utilizado na revisão do VS-MRAC para sistemas com grau relativo unitário. O Lema 6 é aplicado na prova de estabilidade do controlador baseado num observador de alto ganho. O Lema 7 é aplicado na função de modulação do controle a estrutura variável.

No que segue, o termo $\pi_{1}(t)$ é um sinal escalar exponencialmente decrescente, i.e., $\left|\pi_{1}(t)\right| \leq R e^{-\lambda t}, \forall t \geq 0$, para algumas constantes reais $R \geq 0$ e $\lambda>0$. Usa-se a abreviatura "LI" para denotar localmente integrável segundo Lebesgue.

\section{Lema 5 Considere o sistema SISO}

$$
e(t)=M(s)\left[U+d_{U}(t)+\pi_{1}(t)\right],
$$

onde $M(s)$ é uma função de transferência SPR. Assume-se que o sinal $d_{U}(t)$ é LI e o sinal $\pi_{1}(t)$ é LI e exponencialmente decrescente. Se

$$
\begin{aligned}
U & =-\rho(t) \operatorname{sgn}(e), \\
\rho(t) & \geq \delta+\left|d_{U}(t)\right|, \quad \forall t \geq 0,
\end{aligned}
$$

onde $\rho$ é LI e $\delta \geq 0$ é uma constante arbitrária, então $\exists c_{1}, c_{2}, \lambda_{1}>0$ tais que a desigualdade

$$
|e(t)| e\left\|x_{e}(t)\right\| \leq\left[c_{1}\left\|x_{e}(0)\right\|+c_{2} R\right] e^{-\lambda_{1} t}
$$

é satisfeita $\forall t \geq 0$, onde $x_{e}$ é o estado de qualquer realização estabilizável e detectável de (61) (possivelmente nãomínima). Além disso, se $\delta>0$, então e $(t)$ se torna identicamente nulo após algum tempo finito $t_{s} \geq 0$.

Prova: A prova segue (Hsu and Costa, 1989).

Lema 6 Considere o sistema SISO que tem relação entradasaída dada por

$$
\sigma(t)=\frac{K_{p}}{(s+\gamma)}\left[U+d_{U}(t)\right]+\pi_{1}(t)+\beta(t),
$$

e o sinal $\hat{\sigma}(t)=\sigma(t)-\pi_{1}(t)-\beta(t)$, onde $K_{p}, \gamma>0$ são constantes escalares, $\hat{\sigma}, \sigma, U, d_{U}, \beta, \pi_{1} \in \mathbb{R}, d_{U}(t) e ́\left(L I, \pi_{1}(t) e ́\right.$ exponencialmente decrescente e os sinais $\pi_{1}(t)$ e $\beta(t)$ são absolutamente contínuos, $\forall t \geq 0$. Se

$$
U=-\rho(t) \operatorname{sgn}(\sigma),
$$

onde $\rho$ é LI e satisfaz

$$
\rho(t) \geq\left|d_{U}(t)\right|, \quad \forall t \geq 0,
$$

então, os sinais $\sigma(t)$ e $\hat{\sigma}(t)$ são limitados por

$$
|\sigma(t)| \text { e }|\hat{\sigma}(t)| \leq|\hat{\sigma}(0)| e^{-\gamma t}+2\left[R e^{-\lambda_{1} t}+\left\|\beta_{t}\right\|_{\infty}\right],
$$

onde $\lambda_{1}<\min (\gamma, \lambda)$.

Prova: Vide prova do Lema 2 em (Hsu et al., 1997).

Lema 7 Considere um sistema com matriz $p \times m$ de funções de transferência $W(s)$. Seja

$$
\gamma_{0}=\min _{j}\left\{-\Re\left(p_{j}\right)\right\}
$$

a margem de estabilidade de $W(s)$, onde $\left\{p_{j}\right\}$ são os pólos de $W(s)$ e seja $\gamma_{d}:=\gamma_{0}-\delta$ com $\delta>0$ sendo uma constante arbitrária. Seja $\bar{d}(t)$ um majorante instantâneo para o sinal $d(t)$, i.e., $\|d(t)\| \leq \bar{d}(t), \forall t \geq 0$. Então, $\exists c_{1}>0$ tal que a resposta impulsiva $w(t)$ do sistema satisfaça $\|w(t)\| \leq c_{1} e^{-\gamma_{d} t}$ e se verifica a desigualdade

$$
\|w(t) * d(t)\| \leq c_{1} e^{-\gamma_{d} t} * \bar{d}(t)=\frac{c_{1}}{s+\gamma_{d}} * \bar{d}(t), \quad \forall t \geq 0 .
$$

Prova: Vide prova do Lema 2 em (Hsu et al., 2003). 


\section{B PROVA DO TEOREMA 3}

Uma vez que a hipótese (A7) sobre o sinal de controle $u$ é satisfeita, os sinais do sistema podem crescer no máximo exponencialmente. Como se pode representar o vetor regressor $\omega=\Omega_{1} X+\Omega_{2} r$ com matrizes $\Omega_{1}$ e $\Omega_{2}$ adequadas (Hsu et al., 2002, eq. (47)) e lembrando-se que o sinal $r(t)$ é uniformemente limitado, tem-se que $\|\omega\| \leq k_{M}+k_{\Omega}\left\|X_{e}\right\|$. Então, conclui-se que, $\forall t \geq 0$,

$$
\left\|x_{e}(t)\right\| \text { e }\left\|X_{e}(t)\right\| \leq k_{e 1} e^{\lambda_{e 1} t}\left\|X_{e}(0)\right\|+k_{e 2}\left[e^{\lambda_{e 1} t}-1\right] .
$$

Como $t_{e} \leq \bar{t}_{e}$ dado por (48) pode ser majorado por uma função de $\varepsilon$ da classe $\mathcal{K}$, o majorante (71) pode ser majorado $\forall t \in\left[0, t_{e}\right]$ por

$$
\left\|x_{e}(t)\right\| \text { e }\left\|X_{e}(t)\right\| \leq\left(k_{e 3}+k_{e 4} \varepsilon\right)\left\|X_{e}(0)\right\|+\mathcal{K}_{e 6}(\varepsilon),
$$

que, em conjunto com o majorante do erro de estimação do estado (32), permite concluir que, $\forall t \in\left[0, t_{e}\right]$,

$$
\left\|\tilde{x}_{e}(t)\right\| \leq \frac{k_{1}}{\varepsilon^{n^{*}-1}}\left\|\tilde{x}_{e}(0)\right\| e^{-\frac{\lambda_{\alpha}}{\varepsilon} t}+\varepsilon\left[k_{e 9}\left\|X_{e}(0)\right\|+k_{e 10}\right] .
$$

Agora o desenvolvimento de um majorante para o estado $z$ prossegue para $t>t_{1}:=t_{e}$. Tomando-se $t_{1}$ como um novo instante inicial após a extinção do pico no transitório do HGO, tem-se

$$
\begin{aligned}
\left\|\tilde{x}_{e}(t)\right\| & \leq\left[\left\|\tilde{x}_{e}(0)\right\|+\varepsilon\left(k_{e 13}\left\|X_{e}(0)\right\|+k_{e 12}\right)\right] e^{-\lambda_{e}\left(t-t_{1}\right)} \\
& +\varepsilon C\left(t, t_{1}\right), \quad \forall t \geq t_{1} .
\end{aligned}
$$

Reescreve-se a equação do erro (23) como

$$
\dot{x}_{e}=A_{M} x_{e}+B_{M} K\left[U+d_{U}+\pi_{e}(t)\right],
$$

onde $d_{U}:=\left(\theta^{\text {nom }}-\theta^{*}\right)^{T} \omega+W_{d}(s) * d(t)$.

Uma vez que o controle utiliza o estado estimado $\left(\hat{x}_{e}(t)=\right.$ $x_{e}(t)+\tilde{x}_{e}(t)$ ), pode-se reescrever a lei de controle (25) como

$$
\begin{aligned}
U & =-\rho \operatorname{sgn}\left(\sigma\left(\hat{x}_{e}\right)\right), \\
\sigma\left(\hat{x}_{e}\right) & =S x_{e}+S \tilde{x}_{e} .
\end{aligned}
$$

Lembrando-se que $\left\{A_{M}, B_{M}, S\right\}$ é uma realização nãomínima e controlável de $W_{M}(s) L(s)=\frac{K_{M}}{(s+\gamma)}$ e que $K=$ $K_{M}^{-1} K_{p}$, o sinal de chaveamento $\sigma\left(\hat{x}_{e}\right)$ pode ser representado a partir da equação dinâmica (75) e da equação algébrica (77) como

$$
\sigma\left(\hat{x}_{e}\right)=\frac{K_{p}}{(s+\gamma)}\left[U+d_{U}(t)\right]+\pi_{1}(t)+\beta(t),
$$

$\operatorname{com} \pi_{1}(t)+\beta(t)=\frac{K_{p}}{(s+\gamma)} * \pi_{e}(t)+S \tilde{x}_{e}(t)$. Utilizando-se o majorante para $\left\|\tilde{x}_{e}\right\|$ dado por (74), tem-se, $\forall t \geq t_{1}$,

$$
\begin{aligned}
& \left|\pi_{1}(t)\right| \leq k_{e 14}\left[\left\|\tilde{x}_{e}(0)\right\|+\left\|X_{e}(0)\right\|+\varepsilon k_{e 12}\right] e^{-\lambda_{e}\left(t-t_{1}\right)}, \\
& \left\|\beta_{t, t_{1}}\right\|_{\infty} \leq \varepsilon k_{\beta} C\left(t, t_{1}\right) .
\end{aligned}
$$

Então, uma vez que se assumiu que $K_{p}>0$ e a que função de modulação satisfaz $\rho(t) \geq\left|d_{U}(t)\right|(\forall t \geq 0)$, pode-se aplicar o Lema 6 (Apêndice A) ao sistema formado por (78) e pela lei de controle (76), o que resulta no majorante

$$
\begin{aligned}
\left|\sigma\left(\hat{x}_{e}\right)\right| \mathrm{e}\left|\hat{\sigma}\left(\hat{x}_{e}\right)\right| & \leq k_{e 15}\left[\|z(0)\|+\varepsilon k_{e 12}\right] e^{-\lambda_{e}\left(t-t_{1}\right)} \\
& +2 \varepsilon k_{\beta} C\left(t, t_{1}\right), \quad \forall t \geq t_{1}
\end{aligned}
$$

onde $\hat{\sigma}:=\sigma-\pi_{1}(t)-\beta(t)$. Relembrando-se que $u=u^{\text {nom }}+$ $U$, nota-se que $\frac{K_{p}}{(s+\gamma)}$ em (78) opera no mesmo sinal $U+d_{U}$ que aquele em (14). A partir de (78) se conclui que $U+d_{U}=$ $K_{p}^{-1}[\dot{\hat{\sigma}}+\gamma \hat{\sigma}]$. Então, a partir de (14), o erro de rastreamento do modelo pode ser reescrito como

$$
\dot{X}_{e}=A_{c} X_{e}+B_{c} K[\dot{\hat{\sigma}}+\gamma \hat{\sigma}] .
$$

Para eliminar a derivada $\dot{\hat{\sigma}}$, emprega-se a transformação de variáveis $\bar{X}_{e}:=X_{e}-B_{c} K \hat{\sigma}$ resultando em

$$
\dot{\bar{X}}_{e}=A_{c} \bar{X}_{e}+\left(A_{c}+\gamma I\right) B_{c} K \hat{\sigma} .
$$

Uma vez que $A_{c}$ é Hurwitz e que o sinal $\hat{\sigma}$ satisfaz o majorante (81), tem-se $\forall t \geq t_{1}$ que

$$
\left\|\bar{X}_{e}(t)\right\| \leq k_{e 16}\left[\|z(0)\|+\varepsilon k_{e 12}\right] e^{-\lambda_{e}\left(t-t_{1}\right)}+\varepsilon \bar{k} C\left(t, t_{1}\right) .
$$

Além disso, conforme é esclarecido abaixo, $\forall t \geq t_{1}$,

$$
\begin{aligned}
\left\|X_{e}(t)\right\| \mathrm{e}\|e(t)\| & \leq k_{e 17}\left[\|z(0)\|+\varepsilon k_{e 12}\right] e^{-\lambda_{e}\left(t-t_{1}\right)} \\
& +\varepsilon k_{e 18} C\left(t, t_{1}\right) \\
\left\|\omega_{t, t_{1}}\right\|_{\infty} & \leq \varepsilon k_{e 19} C\left(t, t_{1}\right)+k_{e 20}\|z(0)\|+k_{m} \\
C\left(t, t_{1}\right) & \leq \frac{k_{\mathrm{red}}^{\prime}+k_{e 21}\|z(0)\|}{1-\varepsilon k_{e 22}}
\end{aligned}
$$

De fato, as desigualdades em (85) vêm de $X_{e}=\bar{X}_{e}+B_{c} K \hat{\sigma}$. Uma vez que $\|\omega\| \leq k_{M}+k_{\Omega}\left\|X_{e}\right\|$, então, se obtém (86) a partir de (85). Agora, a partir de (33) e (86), pode-se concluir que $C\left(t, t_{1}\right)$ também pode ser majorado por $C\left(t, t_{1}\right) \leq$ $\varepsilon k_{e 22} C\left(t, t_{1}\right)+k_{e 21}\|z(0)\|+k_{\text {red }}^{\prime}$, do qual se obtém por intermédio de manipulações algébricas o majorante (87), que é válido para $\varepsilon<k_{e 22}^{-1}$. Os majorantes (74) para $\left\|\tilde{x}_{e}\right\| \mathrm{e}(85)$ para $\left\|X_{e}\right\|$ podem ser conjugados no majorante

$$
\|z(t)\| \leq\left[k_{e 23}\|z(0)\|+\varepsilon k_{e 24}\right] e^{-\lambda_{e}\left(t-t_{1}\right)}+\varepsilon k_{e 25} C\left(t, t_{1}\right),
$$

$\forall t \geq t_{1}$, no qual pode-se utilizar o majorante (87) para $C\left(t, t_{1}\right)$ resultando em

$$
\begin{aligned}
\|z(t)\| \leq\left[k_{e 23}\|z(0)\|+\varepsilon k_{e 24}\right] e^{-\lambda_{e}\left(t-t_{1}\right)} \\
+\varepsilon \frac{k_{\mathrm{e} 26}+k_{e 27}\|z(0)\|}{1-\varepsilon k_{e 22}}, \quad \forall t \geq t_{1},
\end{aligned}
$$

que para $0<\varepsilon \leq k_{\varepsilon}<\min \left(1, k_{e 22}^{-1}\right)$ pode ser reescrito como

$$
\begin{aligned}
\|z(t)\| & \leq\left[k_{e 23}\|z(0)\|+\varepsilon k_{e 24}\right] e^{-\lambda_{e}\left(t-t_{1}\right)} \\
& +\varepsilon\left[k_{\mathrm{e} 28}+k_{e 29}\|z(0)\|\right], \quad \forall t \geq t_{1},
\end{aligned}
$$


de onde pode-se afirmar que, $\forall t \geq t_{1}$ tem-se

$$
\|z(t)\| \leq\left[k_{e 23} e^{-\lambda_{e}\left(t-t_{1}\right)}+\varepsilon k_{e 29}\right]\|z(0)\|+O(\varepsilon),
$$

onde o termo residual $O(\varepsilon)$ é independente das condições iniciais. Notando-se que o instante inicial é irrelevante na derivação das expressões acima, pode-se escrever

$$
\|z(t)\| \leq\left[k_{e 30} e^{-\lambda_{e}\left(t-t_{i}\right)}+\varepsilon k_{e 31}\right]\left\|z\left(t_{i}\right)\right\|+O(\varepsilon),
$$

para $t \geq t_{i} \geq t_{1}(i=1,2,3, \ldots)$ arbitrário. Esta desigualdade conduz à desigualdade linear recursiva

$$
\left\|z\left(t_{i+1}\right)\right\| \leq \lambda\left\|z\left(t_{i}\right)\right\|+O(\varepsilon),
$$

$\operatorname{com} \lambda=k_{e 30} e^{-\lambda_{e} T_{1}}+\varepsilon k_{e 31}$ e algum período $T_{1}=t_{i+1}-t_{i}>$ 0 . Para $0<\varepsilon \leq \varepsilon^{*}<k_{e 31}^{-1}$ e escolhendo-se o período $T_{1}>0$ adequadamente longo, obtém-se $\lambda<1$. Assim conclui-se que para $\varepsilon>0$ suficientemente pequeno, a recursão (93) converge em progressão geométrica para um conjunto residual de ordem $\varepsilon$.

Conjugando-se a convergência exponencial do estado $z(t)$ para um conjunto residual de ordem $\varepsilon$ a partir do instante $t_{1}$, o que se conclui da recursão (93), aos majorantes (72) para $\left\|X_{e}\right\|$ e (73) para $\left\|\tilde{x}_{e}\right\|$, válidos para $0 \leq t \leq t_{1}$, obtém-se finalmente os majorantes (52) e (53) para as normas dos vetores de estado das equações dos erros que são válidos $\forall t \geq 0$. 